\title{
Fractionalization patterns in strongly correlated electron systems: Spin-charge separation and beyond
}

\section{Citation}

Demler, Eugene, Chetan Nayak, Hae-Young Kee, Yong Baek Kim, and T. Senthil. 2002.

"Fractionalization Patterns in Strongly Correlated Electron Systems: Spin-Charge Separation and Beyond." Physical Review B 65 (15) (March 27). doi:10.1103/physrevb.65.155103.

\section{Published Version}

doi:10.1103/PhysRevB.65.155103

\section{Permanent link}

http://nrs.harvard.edu/urn-3:HUL.InstRepos:27989551

\section{Terms of Use}

This article was downloaded from Harvard University's DASH repository, and is made available under the terms and conditions applicable to Other Posted Material, as set forth at http:// nrs.harvard.edu/urn-3:HUL.InstRepos:dash.current.terms-of-use\#LAA

\section{Share Your Story}

The Harvard community has made this article openly available.

Please share how this access benefits you. Submit a story.

\section{Accessibility}




\title{
Fractionalization patterns in strongly correlated electron systems: Spin-charge separation and beyond
}

\author{
Eugene Demler, ${ }^{1}$ Chetan Nayak, ${ }^{2}$ Hae-Young Kee, ${ }^{2}$ Yong Baek Kim,${ }^{3}$ and T. Senthil ${ }^{4,5}$ \\ ${ }^{1}$ Physics Department, Harvard University, Cambridge, Massachusetts 02138 \\ ${ }^{2}$ Physics Department, University of California Los Angeles, Los Angeles, California 90095-1547 \\ ${ }^{3}$ Department of Physics, The Ohio State University, Columbus, Ohio 43210 \\ ${ }^{4}$ Institute for Theoretical Physics, University of California, Santa Barbara, California 93106-4030 \\ ${ }^{5}$ Massachusetts Institute of Technology, 77 Massachusetts Ave., Cambridge, Massachusetts 02139
}

(Received 22 May 2001; published 27 March 2002)

\begin{abstract}
We discuss possible patterns of electron fractionalization in strongly interacting electron systems. A popular possibility is one in which the charge of the electron has been liberated from its Fermi statistics. Such a fractionalized phase contains in it the seed of superconductivity. Another possibility occurs when the spin of the electron, rather than its charge, is liberated from its Fermi statistics. Such a phase contains in it the seed of magnetism, rather than superconductivity. We consider models in which both of these phases occur and study possible phase transitions between them. We describe other fractionalized phases, distinct from these, in which fractions of the electron themselves fractionalize, and discuss the topological characterization of such phases. These ideas are illustrated with specific models of $p$-wave superconductors, Kondo lattices, and coexistence between $d$-wave superconductivity and antiferromagnetism.
\end{abstract}

DOI: 10.1103/PhysRevB.65.155103 PACS number(s): 71.10.Hf, 74.20.Mn, 71.27.+a, 74.72.-h

\section{INTRODUCTION}

Electron fractionalization in strongly interacting electron systems in dimensions larger than 1 has been an important subject of study since spin-charge separation was suggested as a mechanism of high- $T_{c}$ superconductivity ${ }^{1,2}$ in the cuprates. In particular, it was suggested that the electron is splintered into a spin-carrying neutral excitation ("spinon") and a charge-carrying spinless excitation ("holons" or "chargons"). There have been different proposals in regard to this possibility, but the existence of such phases in the cuprates is still controversial.

On the other hand, there exist clear experimental examples of phases in the quantum Hall regime of twodimensional electron systems where quantum number fractionalization has been well established. The low-energy excitations (quasiparticles) in these two-dimensional strongly interacting electron systems carry fractions of the quantum numbers of the original electrons. Different quantum Hall liquid states can be characterized by different varieties of topological order. The transitions between different quantum Hall states can be understood as topological-order-changing transitions which occur even in the absence of conventional broken symmetries. The Hall conductance is but one of the topological quantum numbers which characterize a given phase. Another important property of a topologically ordered state is the ground-state degeneracy of the system on higher genus manifolds such as tori. For each topologically ordered state, there are corresponding sets of characteristic excitations with different quantum numbers.

It has become clear ${ }^{3,4}$ that the notion of topological order also provides a precise characterization of spin-charge separated and other fractionalized phases in spatial dimensions higher than one even in situations of zero or weak magnetic fields. One of the remarkable features of the quantum Hall effect is the enormously rich number of exotic phases which display different patterns of fractionalization of the electron and associated topological orders. In view of the similarity between the theoretical characterization of quantum Hall states and fractionalized states in zero magnetic field, it is tempting to investigate a similar possibility of a variety of fractionalization patterns in other strongly correlated systems. We explore this possibility in this paper. We describe theoretically a few of the several different possible fractionalized phases that may exist in various different models of strongly interacting electron systems.

Following the introduction of the Schwinger boson description of the Heisenberg model of quantum antiferromagnets, ${ }^{5}$ slave fermion ${ }^{6}$ formulations of doped antiferromagnets were introduced. In these formulations, it is assumed that the electron decays into a Bosonic, spin-1/2 spinon and a fermionic, charge-e holon. We will call this phase CFSB (charged fermion, spinful boson).

On the other hand, a phase with Bosonic holons and fermionic spinons - which we will call CBSF (charged boson, spinful fermion)-naturally leads to superconductivity through the Bose condensation of Bosonic holons in the presence of Fermionic spinon pairing. Consequently, much attention has been focused on the description of such a fractionalized phase, especially in the context of the slave boson description of the $t-J$ model. The pairing symmetry of the resulting superconductor is dictated by the underlying symmetry of the spinon pairing.

$\mathrm{A} Z_{2}$ gauge theory of Fermionic spinons and Bosonic holons was developed in the context of superconductivity in the cuprates $^{7}$ (see also Refs. 8-10). Spinons and holons are coupled by an Ising gauge field. The deconfined phase of this theory corresponds to the CBSF phase. Most importantly, the deconfinement-confinement transition of spinons and holons occurs through the condensation of vortices in the $Z_{2}$ gauge 
field, or visons. In the deconfined phase, the visons exist as gapped excitations; when visons condense, the spinons and holons are confined within electrons. The existence of gapped visons is crucial for the robustness of the topological order of the deconfined fractionalized phase. ${ }^{11,4}$ Although this formalism was introduced in the context of cuprate superconductivity, it is sufficiently flexible to permit a description of other types of fractionalized phases including CFSB.

These ideas have a physical manifestation in the context of quantum disordered magnets and superconductors. In this picture, one visualizes fractionalized states in terms of nearby ordered states. In a broken (continuous) symmetry state, Goldstone modes can screen the associated quantum number(s). ${ }^{12}$ Thus it is possible for quasiparticles to be stripped of some of their quantum numbers. One might imagine that the destruction of order by quantum fluctuations can preserve this screening of quasiparticle quantum numbers. This occurs when those topological defects of the ordered state which braid nontrivially with the quasiparticles persist as gapped excitations even after the demise of the order. ${ }^{13}$ Indeed, this is precisely what happens when the state is topologically ordered. The neutral, spin- $1 / 2$ fermion of the CBSF state is viewed as the descendent of the Bogoliubov-de Gennes quasiparticle; the vison, of the $h c / 2 e$ vortex. When considered in the context of spin-triplet superconductors and their rich order-parameter structure, this immediately suggests exotic phases such as CBSF, CFSB, and even a third phase CBSBNF (charged boson, spinful boson, neutral fermion), in which the charge- and spin-carrying excitations are bosons and there is a neutral, spinless Fermionic excitation. Since these superconductors can break both charge and spin symmetries - as do states in which singlet superconductivity and magnetism coexist - one can envision the screening of both quantum numbers of a quasiparticle. If (the minimal) topological defects in the charge sector survive into a disordered state, then this disordered state has neutral, spin-1/2 Fermionic excitations (CBSF); if topological defects in the spin sector survive into a disordered state, then this disordered state has charge-e spinless Fermionic excitations (CFSB); if topological defects in the both sectors survive into a disordered state, then this disordered state has neutral, spinless Fermionic excitations (CBSBNF).

The analysis of quantum dimer models ${ }^{2}$ and resonating valence-bond $^{1,14,15}$ ground states led to conflicting claims that the CBSF (Ref. 15) or CFSB (Refs. 16 and 17) scenario is realized in these models. These models have a $Z_{2}$ vortex excitation ${ }^{17,18}$ - which are precisely the visons described above - which are relative semions with spinons and holons. Thus a spinon or holon can change between Bosonic and Fermionic statistics by forming a bound state with a vison. This begs the question whether the CBSF phase discussed in the context of superconductivity is the same as the CFSB phase considered in relation to magnetism. We reconsider this question in the context of recent progress in the understanding of fractionalized phases described above. One might worry that the apparent differences between these phases is an artifact of the formalisms employed. One might also wonder if there are any further fractionalized phases. In this paper, we discuss the questions raised above using three different models: $p$-wave superconductors, Kondo lattices, and $X Y$ magnets coupled to $d$-wave superconductors.

The main results can be summarized as follows.

(i) Both CBSF and CFSB phases can arise in a variety of different models.

(ii) Upon accepting the possibility of electron fractionalization, one is led to consider a wide variety of fractionalized phases. In the higher-level fractionalized phases, electrons can be fractionalized in many different ways. For example, spinons and holons can be further fractionalized. Apart from the CBSF and CFSB phases, we discuss two others. One is the CSBNF (charge- and spin-carrying boson, neutral fermion) phase, in which the electron breaks up into a boson which carries both the spin and charge quantum numbers and a neutral fermion. This phase is at the first level of fractionalization along with the CBSF and CFSB phases. The other is the CBSBNF phase, in which there exist spin-carrying neutral bosons, charge-carrying spinless bosons, and "statistics-carrying" neutral spinless fermions. The CBSBNF phase is at the second level of fractionalization. In principle, higher-level fractionalized phases exist.

(iii) We demonstrate the existence of some of these exotic phases in the context of the three different systems mentioned above-Kondo lattices, $p$-wave superconductors, and models with both strong spin and $d$-wave pairing fluctuations. For the $p$-wave superconductor, the four fractionalized phases discussed here arise naturally and the order parameter has a rich spectrum of topological defects which can condense in a variety of ways, thereby giving rise to an array of fractionalized nonsuperconducting phases.

(iv) The question of whether CBSF and CFSB are smoothly connected to one another or whether they are necessarily separated by a phase transition is a subtle and delicate issue for reasons that will be discussed at length later. While we do not provide a definitive conclusion, we outline a possible scenario in which the distinction between CBSF and CFSB is similar to that between liquid and gas phases. These phases are separated by a first-order transition line which terminates at a critical point. In principle, one can go around the critical point from one phase to the other without encountering a phase transition. This scenario is supported by a number of suggestive (though certainly not conclusive) arguments.

On the other hand, the transition between the two phases can occur through another fractionalized phase with a higherlevel fractionalization pattern. In this case, each transition in the process could be a continuous transition. We demonstrate that the transition between CBSF phase and CFSB phase can occur through the CBSBNF phase.

(v) In order to examine whether one can go from CBSF to CFSB through further fractionalized phases like CBSBNF, one can design a gedanken flux trapping experiment similar to the one proposed in Ref. 19. This gedanken experiment clearly demonstrates the existence of a phase boundary between CBSF and CFSB when these phases are close to CBSBNF.

Topological order is robust against local perturbations such as impurities. Thus we will concentrate on general universal properties of the fractionalized phases. One of our 
goals will be to give a precise characterization of these phases which is independent of the underlying microscopic models where they may occur. We believe that these exotic phases could play a role in the physics of ${ }^{3} \mathrm{He}$ (Ref. 20) and the ruthenates ${ }^{21}$ as well as the cuprates, ${ }^{22}$ organic superconductors, ${ }^{23}$ heavy fermion superconductors, ${ }^{24}$ spinor Bose-Einstein condensates, ${ }^{25}$ and the crusts of neutron stars. $^{26}$

The rest of the paper is organized as follows. In Sec. II, we consider a Kondo lattice model and how the CFSB fractionalized phase can occur in this model using the language of a $Z_{2}$ gauge theory. Some details are given in Appendix A. In Sec. III, we suggest how this analysis can be generalized and discuss a hierarchy of fractionalized phases. Here we provide an overview of our results. In Sec. IV, we discuss how this hierarchy can be realized in $p$-wave superconducting systems when the superconducting and spin order are quantum disordered. This is done using the vortex condensation formalism. In Appendix B, the same ideas are shown to apply to an $X Y$ magnet which is coupled to a $d$-wave superconductor. In Sec. V, the fractionalized phases of Sec. IV are further discussed in the framework of a $Z_{2} \times Z_{2}$ gauge theory. In Sec. VI, we consider the question of the distinction in principle between the putatively different fractionalized phases constructed in this paper. In Appendix $\mathrm{C}$, we give some technical details of an argument using $Z_{2} \times Z_{2}$ gauge theory which supports our picture of the phase diagram. In Sec. VII, we show how flux-trapping experiments (of the variety suggested by Senthil and Fisher ${ }^{19}$ ) can be used to shed further light on the phase boundaries between these phases and could be used to detect them. We conclude in Sec. VII. Appendix D contains an aside in which we discuss various interesting properties of unfractionalized phases occurring in the models considered in this paper.

For other perspectives on fractionalization, see Refs. $1-4,7,8,12,13,15,17-19$, and $27-33$.

\section{FRACTIONALIZATION IN SPIN MODELS: SPIN-STATISTICS SEPARATION}

In principle, there are several possible ways in which the electron can fractionalize in a strongly correlated system. In the context of the cuprates, attention has been focused on the situation in which the electron splinters into two separate excitations - a charged spinless boson, and a neutral spinful fermion. In this case, the charge of the electron is liberated from its Fermi statistics.

In this section, we will briefly discuss another possible fractionalization pattern in which the spin, rather than the charge, of the electron is liberated from its Fermi statistics. The electron splinters into a charged spinless fermion, and a spinful boson. As we will see, this phenomenon also requires the presence of a gapped topological $Z_{2}$ vortex excitation. The issue of whether such a fractionalized phase is distinct from one in which the charge is liberated from the Fermi statistics is a delicate one, and shall be discussed in Sec. VI.

To motivate the discussion, consider a "Kondo lattice" model with the Hamiltonian

$$
H=H_{t}+H_{K}+H_{e x},
$$

$$
\begin{gathered}
H_{t}=-\sum_{\left\langle r r^{\prime}\right\rangle} t_{r r^{\prime}}\left(c_{r \alpha}^{\dagger} c_{r^{\prime} \alpha}+\text { H.c. }\right), \\
H_{K}=J_{K} \sum_{r}\left(S_{r}^{+} c_{r \downarrow}^{\dagger} c_{r \uparrow}+\text { H.c. }\right), \\
H_{e x}=\sum_{r r^{\prime}}-\frac{J}{2}\left(S_{r}^{+} S_{r^{\prime}}^{-}+\text {H.c. }\right)+J_{z} S_{r}^{z} S_{r^{\prime}}^{z} .
\end{gathered}
$$

Here the $c_{i \alpha}$ represent "conduction" electrons with spin $\alpha$ at site $i$. The operators $\vec{S}_{i}$ are spin operators representing magnetic moments localized at the lattice sites. The first term is the usual conduction electron hopping, described in a tightbinding approximation. The second term is a "Kondo" coupling between the conduction electrons and the local moments. The third term is an explicit exchange interaction between the local moments. For simplicity, we have assumed that system only has a U(1) spin symmetry for rotations about the $z$ axis of spin [we will comment on situations with full $\mathrm{SU}(2)$ spin symmetry later]. We are interested not so much in establishing the exact phase diagram of this particular model; rather our main interest here is in establishing the possible existence and stability in models of this kind of quantum phases where the electron is fractionalized. To that end, we will think more generally about a class of models which may be obtained from the model above by adding other local interactions which share its symmetries. If the system is in a quantum phase in which both the symmetry of rotations about the $z$ direction of spin and the charge conservation symmetry is unbroken, the excitations may be labeled by their $S_{z}$ and charge $(Q)$ quantum numbers. Clearly, we can visualize two qualitatively different possibilities. First, the system may be in a phase in which the excitations are electrons $\left(Q=1, S_{z}=\frac{1}{2}\right)$ or composite objects made from electrons (such as, for instance, a magnon which has $Q=0, S_{z}$ $=1$ ). This is a conventional phase of the kind familiar from textbooks (for instance, a Fermi liquid or a band insulator). On the other hand, one could also imagine phases in which there are excitations which carry quantum numbers which are fractions of those of an electron. The simplest possibility (the one we will focus on) is that there are excitations which carry $S_{z}=1 / 2, Q=0$ (spinons) and others which carry $S_{z}$ $=0, Q=1$ (holons). In such a phase, the electron has been fractionalized. In what follows, we will discuss several ways of thinking about such phases. Our focus will be on general universal properties of such phases. In particular, we will be interested in obtaining robust precise characterizations of fractionalized phases that are independent of the particular microscopic models in which they possibly occur.

It is extremely instructive to begin by just considering the physics of the local moments alone as described by the exchange part of the Hamiltonian $H_{e x}$. This Hamiltonian is clearly invariant under a global spin rotation about the $z$ axis of spin. For technical simplicity, we will assume $J, J_{z} \geqslant 0$. The physics of this particular Hamiltonian is well understood: when $J_{z} / J$ is small, there is long-range order in $S^{+}$. When $J_{z} / J$ is large, the system breaks translational symmetry with $\left\langle S^{z}\right\rangle$ being larger in one sublattice of the square 
lattice than the other, but the $\mathrm{U}(1)$ spin rotation symmetry is unbroken. The point $J_{z}=J$ can be mapped to the nearestneighbor antiferromagnetic Heisenberg model with full SU(2) spin symmetry on a bipartite lattice by rotating the spins on one sublattice by $\pi$ about the $z$ axis. In the specific case of a square lattice (which we assume through out our discussion), this is known to develop Néel long-range order in two spatial dimensions. Our interest here is not so much in the properties of this particular Hamiltonian as in the properties of an entire class of systems with the same symmetry, and with short-ranged interactions between the spins. In particular, we will be interested in fractionalized phases in which the excitations are spinons with quantum number $S_{z}$ $=1 / 2$. To that end, we will reformulate the Hamiltonian directly in terms of spinon fields which carry spin $S^{z}=1 / 2$. This naturally introduces a $Z_{2}$ gauge symmetry. The result is a theory of Bosonic spinon fields coupled to a $Z_{2}$ gauge field which can then be used to analyze the possibility of fractionalized phases and their universal properties.

We may think of $S^{+}, S^{-}$as the creation and destruction operators, respectively, of a hard-core boson on the sites of the lattice. Specifically, write $S_{r}^{+} \equiv b_{s r}^{\dagger}, S_{r^{\prime}}^{-} \equiv b_{s r}$, and $S_{r}^{z}$ $=1 / 2-b_{s r}^{\dagger} b_{s r}$. Note that there is half a boson for each site on average. Now imagine relaxing the hard-core constraint on the bosons, and instead add a term

$$
\frac{U}{2} \sum_{r}\left(2 n_{r}-1\right)^{2}
$$

at each lattice site. Here $n_{r}$ is the boson number at each site. In the limit $U \rightarrow \infty$, we recover the spin model exactly. For large but finite $U$, however, relaxing the hard-core constraint is expected to be innocuous. It is now convenient to go to a number-phase representation for the bosons: we write $b_{s r}$ $\sim e^{i \varphi_{r}}$ with $\left[\varphi_{r}, n_{r^{\prime}}\right]=i \delta_{r r^{\prime}}$. For simplicity, we also specialize to the limit where $J_{z}=0$. The Hamiltonian then becomes

$$
H=\sum_{\left\langle r r^{\prime}\right\rangle}-J \cos \left(\varphi_{r}-\varphi_{r^{\prime}}\right)+\frac{U}{2} \sum_{r}\left(1-2 n_{r}\right)^{2} .
$$

This is clearly closely related to the original spin Hamiltonian in Eq. (4). Now consider a formal change of variables which involves splitting the boson operator $b_{s r}$ into two pieces:

$$
\begin{aligned}
& b_{s r}=e^{i \varphi_{r}}=z_{r}^{2}, \\
& z_{r} \equiv e^{i \phi_{r}}=s_{r} e^{i\left(\varphi_{r} / 2\right)} \quad\left(s_{r}= \pm 1\right) .
\end{aligned}
$$

We will refer to $z_{r}$ as the spinon destruction operator. Note that with these definitions, both $\varphi_{r}$ and $\phi_{r}$ are defined in the interval $[0,2 \pi)$. It is also convenient to define a number operator for the spinons $N_{r}=2 n_{r}$ which is conjugate to $\phi_{r}$. In terms of the spinon operator, the Hamiltonian becomes

$$
H=\sum_{\left\langle r r^{\prime}\right\rangle}-J \cos \left(2 \phi_{r}-2 \phi_{r^{\prime}}\right)+\frac{U}{2} \sum_{r}\left(N_{r}-1\right)^{2} .
$$

The change of variables above must be supplemented with a constraint-clearly the physical Hilbert space consists only of states where $N_{r}$ is even. Therefore we need to impose the operator constraint $(-1)^{N_{r}}=1$ at each site of the lattice. Formally this may be implemented through the projection operator

$$
\begin{gathered}
\mathcal{P}=\prod_{r} \mathcal{P}_{r}, \\
\mathcal{P}_{r}=\frac{1}{2}\left[1+(-1)^{N_{r}}\right] .
\end{gathered}
$$

Note that $[\mathcal{P}, H]=0$. It is now convenient to pass to a functional integral formulation. We follow Refs. 7 and 27 closely to obtain for the partition function

$$
\begin{gathered}
Z=\sum_{\sigma_{r \tau}} \int \mathcal{D} \phi e^{-S}, \\
S=S_{\tau}+S_{r}+S_{B}, \\
S_{\tau}=\sum_{\tau, r} J_{\tau} \sigma_{r \tau} \cos \left(\phi_{r, \tau+\epsilon}-\phi_{r \tau}\right), \\
S_{r}=\epsilon \sum_{\left\langle r r^{\prime}\right\rangle \tau} J \cos \left(2 \phi_{r \tau}-2 \phi_{r^{\prime} \tau}\right),
\end{gathered}
$$

where $\sigma_{r \tau}= \pm 1$ may be interpreted as the time component of a $Z_{2}$ gauge field that imposes the constraint on the Hilbert space, and $\epsilon$ is the lattice spacing along the time direction. The constant $J_{\tau}$ is determined by the original interaction strength $U$. The term in the action $S_{r}$ involving the spatial coupling may be decoupled by a Hubbard-Stratanovich transformation:

$$
e^{-S_{r}}=\int D \chi e^{-\epsilon J \Sigma_{\left\langle r r^{\prime}\right\rangle, \tau} \chi_{r r^{\prime}}(\tau)^{2}+2 \epsilon J \chi_{r r^{\prime}}(\tau)\left[z_{r}^{\dagger}(\tau) z_{r^{\prime}}(\tau)+\text { c.c. }\right]} .
$$

Here $\chi_{r r^{\prime}}(\tau)$ is a real-valued field. We have omitted an unimportant overall constant.

We now proceed exactly as in Refs. 7 and 27, and replace the integral over the continuous variable $\chi$ by a sum over a discrete field $\sigma_{r r^{\prime}}(\tau)= \pm 1$. As discussed in Refs. 7 and 27, this approximation respects all the symmetries of the action, and is expected to be innocuous. The resulting partition function becomes

$$
\begin{gathered}
Z=\sum_{\sigma_{i j}} \int \mathcal{D} \phi e^{-S}, \\
S=S_{s}+S_{B}, \\
S_{s}=-\sum_{\langle i j\rangle} J_{i j} \sigma_{i j} \cos \left(\phi_{i}-\phi_{j}\right) .
\end{gathered}
$$

Here the $i, j$ label the sites of a space-time lattice in three dimensions. The constants $J_{i j}=J_{\tau}$ for temporal links, and equals $\epsilon J$ for spatial links. $S_{B}$ is the Berry phase action 


$$
S_{B}=\frac{\pi i}{2} \sum_{i, j=i+\hat{\tau}}\left(1-\sigma_{i j}\right) .
$$

Note that the action (18)-(20) respects all the symmetries of the original model. The discrete field $\sigma_{i j}= \pm 1$ may be viewed as a $Z_{2}$ gauge field. At this stage, this field does not have any dynamics. However, it is natural to expect that upon coarse graining, some dynamics will be generated. The simplest such term allowed by symmetry is

$$
S_{K}=-K \sum_{\square} \prod_{\square} \sigma_{i j}
$$

We will therefore consider the full action

$$
S=S_{S}+S_{K}+S_{B}
$$

What we have achieved so far is an approximate reformulation of spin models with $X X Z$ symmetry. This reformulation is extremely useful to explore the various possible allowed phases in such models. However, the approximations made in obtaining this reformulation are severe enough that it is not easy to see which one of these allowed phases will be obtained in any particular microscopic model.

Consider the possible phases when the parameter $K$ is very large. When $K=\infty$, the $Z_{2}$ flux through each plaquette is constrained to be 1 . We may then choose a gauge in which $\sigma_{i j}=1$ on every link. In this limit therefore, the action reduces to

$$
S=-\sum_{\langle i j\rangle} J_{i j} \cos \left(\phi_{i}-\phi_{j}\right)
$$

This simply describes a quantum $X Y$ model in two spatial dimensions. Note that the Berry phase term simply vanishes when all the $\sigma_{i j}=1$. There clearly are two possible phases - an $X Y$ ordered phase in which $z_{i}=e^{i \phi_{i}}$ has condensed, and a paramagnetic phase in which the excitations created by $z_{i}$ are gapped. Note that these excitations in the paramagnetic phase carry spin $S^{z}=1 / 2$. Thus the spin has been fractionalized in this phase.

Now consider moving away from the limit $K=\infty$ by making $K$ large but finite. For finite $K$, as can be seen from the arguments advanced in Ref. 7, the $X Y$ ordered phase where the spinon field has condensed is indistinguishable from a conventional $X Y$ ordered $X X Z$ magnet. The paramagnetic phase in which the spinons are uncondensed and deconfined survives for large but finite $K$. When $K$ is finite, it becomes clear that this phase has another distinct excitation which carries the flux of the $Z_{2}$ gauge field. This $Z_{2}$ vortexdubbed the vison-does not carry any physical spin, and has an energy gap of order $K$ for large $K$. It has the important property that when a spinon is taken around it, the wave function of the system acquires a phase of $\pi$.

Upon decreasing $K$, at some critical value, the vison gap goes to zero. For smaller $K$, the visons condense leading to confinement of the spinons. The resulting phase is a conventional quantum paramagnet with gapped $S_{z}=1$ excitations. In this phase, the Berry phase term becomes important and leads to a breaking of translational symmetry - the paramag- net is therefore expected to develop spin-Peierls order. We will not discuss such conventional phases very much in this paper.

Much further insight into the physics of the fractionalized phases may be obtained by the following considerations. We begin by first considering ordered phases in which the symmetry of rotations about the $z$ direction of spin has been broken spontaneously. For simplicity, we consider a phase in which the spins have all lined up along some direction in the $x y$ plane. The general properties of such a phase are well known. There are two distinct kinds of excitations. First, there is a gapless spin-wave mode with linear dispersion. Apart from these, there are also topological vortex excitations. On moving along any circuit that encloses a vortex, the direction of the spin in the $x y$ plane winds by an integer multiple of $2 \pi$. This integer winding number-the vorticity - is conserved, and may be used to label the spectrum of excited states. States with different total vorticity belong to different topological sectors and are not mixed by the dynamics generated by the Hamiltonian. Note that in this ordered phase we can no longer label states by their $S^{z}$ quantum number.

These familiar properties of the $X Y$ ordered phase must be contrasted with those of the quantum paramagnet. First consider a conventional paramagnet (i.e., one with no fractionalization). Clearly in this phase $S^{z}$ is conserved, and is a good quantum number to label the excitation spectrum. On the other hand, the vorticity loses its meaning in the paramagnetic phase, and is no longer a good quantum number. This suggests that one may view the paramagnet as a phase in which the vortex excitations have themselves condensed. Condensation of the vortices implies that the vorticity is no longer a good quantum number (just like condensation of spin implies that $S^{z}$ is no longer a good quantum number). Indeed, these observations may be formalized precisely by means of a duality transformation which reformulates the system in terms of the vortex fields rather than the spins. In this dual formulation, the paramagnet is described as a vortex condensate, and the $X Y$-ordered phase as a vortex insulator (in which the vortices are gapped). The physical excitations of the paramagnet which carry the $S^{z}$ quantum number appear as dual flux tubes of the vortex condensate in this language.

How are we to view the fractionalized quantum paramagnet in this dual language? As the phase in question is a paramagnet, it is clear that the vorticity has no meaning, implying that the vortices must have condensed. As pointed out in Ref. 13, we may view the fractionalized phase as a condensate of paired vortices. This has the immediate consequence of halving the dual flux tube, i.e., of fractionalizing $S^{z}$ as required. Furthermore, note that the unpaired (and uncondensed) single vortex is still an excitation in the system. Its vorticity is screened by the (double strength) vortex condensate as is required in the paramagnet. However, its parity is still a good quantum number. Thus the unpaired vortex, though a legitimate excitation of the fractionalized paramagnet, carries only a $Z_{2}$ quantum number-it is clear that it is the vison excitation discussed previously. 
The discussion above provides a description of a fractionalized quantum paramagnet in the context of spin models with $X X Z$ symmetry. We now return to the full model which includes coupling to the "conduction" electron degrees of freedom. As above, we first replace the operator $S_{r}^{-}$in the Kondo coupling at each site by the boson operators $b_{s r}$ $\sim e^{i \varphi_{r}}$ (and similarly for $S_{r}^{+}$). The Kondo coupling term then becomes

$$
\begin{aligned}
H_{K} & =J_{K} \sum_{r}\left(b_{s r}^{\dagger} c_{r \downarrow}^{\dagger} c_{r \uparrow}+\text { H.c. }\right) \\
& =J_{K} \sum_{r}\left(z_{r}^{2 \dagger} c_{r \downarrow}^{\dagger} c_{r \uparrow}+\text { H.c. }\right) .
\end{aligned}
$$

In going to the second equation, we have introduced the spinon operators $z_{r}$ defined in Eq. (7). The Kondo coupling can be further simplified by another change of variables,

$$
\begin{gathered}
\eta_{r \uparrow} \equiv z_{r} c_{r \uparrow}, \\
\eta_{r \downarrow} \equiv z_{r}^{\dagger} c_{r \downarrow} .
\end{gathered}
$$

We will call the $\eta$ operators the holon operators. In terms of the holons, the Kondo coupling becomes

$$
H_{K}=J_{K} \sum_{r}\left(\eta_{r \uparrow}^{\dagger} \eta_{r \downarrow}+\text { H.c. }\right) .
$$

Note that the holons are actually spinless charge $e$ fields despite the presence of the label $\uparrow, \downarrow$. This is obvious from their definition in terms of the spinon and electron operators above: the holon operators do not transform under spin rotations about the $z$ axis. Explicitly, the Kondo term mixes up and down holons so that their label $\uparrow, \downarrow$ is changed by the dynamics. Therefore their spin label has no great significance, and they are correctly viewed as spinless fermions. We may use the following physical picture: the Kondo spins screen the spin of the conduction electrons.

Under these changes of variables, the electron hopping term becomes

$$
H_{t}=-\sum_{\left\langle r r^{\prime}\right\rangle} t_{r r^{\prime}}\left[z_{r}^{\dagger} z_{r^{\prime}}\left(\eta_{r^{\prime} \uparrow}^{\dagger} \eta_{r^{\prime} \uparrow}+\eta_{r \downarrow}^{\dagger} \eta_{r^{\prime} \downarrow}\right)+\text { H.c. }\right] .
$$

We now make approximations very similar to those used above for the exchange part of the Hamiltonian. They allow us to reformulate the system in terms of the spinons, holons, and a $Z_{2}$ gauge field. Some of the details are outlined in the Appendix. The resulting action can essentially be guessed on symmetry grounds, and takes the form

$$
\begin{gathered}
S=S_{c}+S_{s}+S_{B}+S_{K}, \\
S_{c}=-\sum_{\langle i j\rangle} \sigma_{i j} t_{i j}^{c}\left(\eta_{i \uparrow}^{\dagger} \eta_{j \uparrow}+\eta_{i \downarrow}^{\dagger} \eta_{j \downarrow}+\text { c.c. }\right) \\
+J_{K} \sum_{i}\left(\eta_{i \uparrow}^{\dagger} \eta_{j \downarrow}+\text { c.c. }\right) .
\end{gathered}
$$

The other terms of the action are as given before.

Following the discussion above, for large $K$, we expect to have a phase in which the holons and spinons are liberated from each other. In such a phase, the electron is fractionalized. However, in contrast to the fractionalized phase that is most popular in the context of the cuprates, here the spin of the electron has been liberated from its Fermi statistics. Are these two phases actually the same? We will address this issue in subsequent sections.

Though we have based our discussion on models with $X X Z$ symmetry, we expect the fractionalized quantum paramagnetic phase to exist even in systems with full SU(2) spin symmetry. Indeed, in the context of frustrated quantum $S p(n)$ spin models in the large- $n$ limit, Read and Sachdev ${ }^{8}$ have argued for the stability of fractionalized paramagnetic phases with properties similar to that discussed above.

\section{A HIERARCHY OF FRACTIONALIZED PHASES}

In Sec. II, we primarily discussed fractionalized phases in which the electron splinters into a spin-1/2 neutral boson and a charged spinless fermion. For future convenience, we will refer to this as the CFSB (charged fermion, spinful boson) phase. This is to be contrasted with the fractionalized phases which are popular in the context of cuprate physics in which the electron splinters into a spin-1/2 neutral fermion and a charged spinless boson (see also Secs. IV and V). We will refer to this as the CBSF phase. In both cases, there is, in addition, a $Z_{2}$ vortex excitation (the vison) such that taking either the holon or spinon around it produces a phase change of $\pi$.

Having accepted the possibility of quantum number frac-

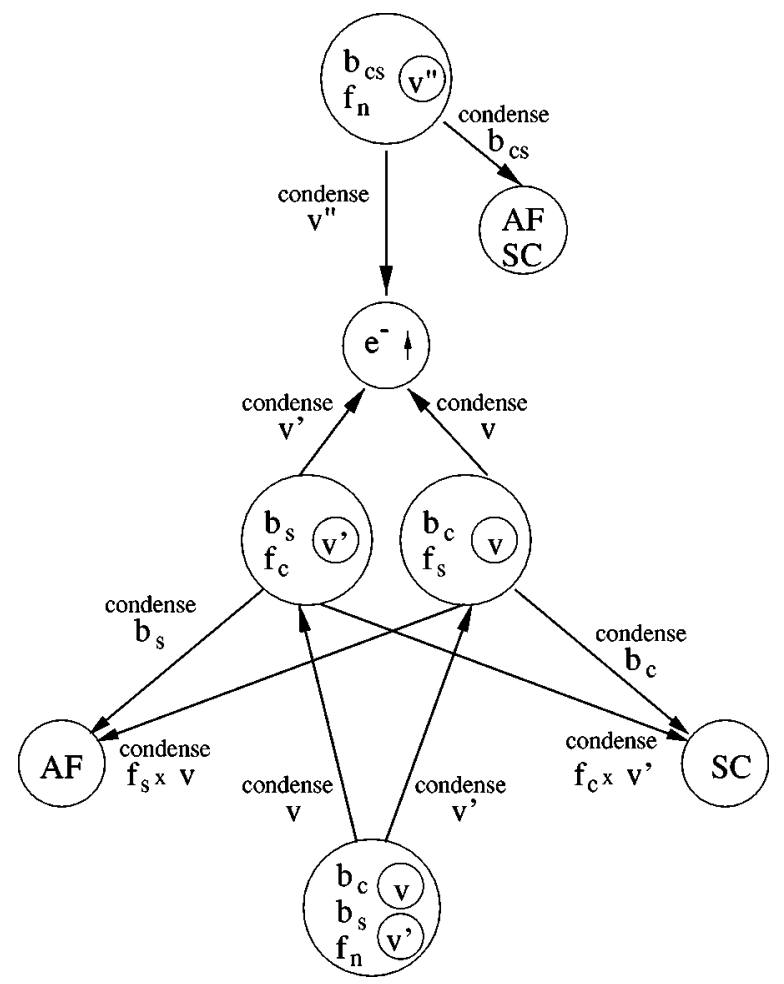

FIG. 1. Hierarchy of fractionalized phases. 
tionalization, one can imagine a wide variety of possible phases apart from the two mentioned above. In particular, one may consider exotic possibilities where the fractions of the electron in any given fractionalized phase themselves fractionalize. Such phases may be considered to have a higher level of fractionalization. To see how these may be described in the same kind of formulation as discussed in this section, consider the following action:

$$
\begin{gathered}
S=S_{f}+S_{c}+S_{s}+S_{\sigma \tau}, \\
S_{f}=-\sum_{i j, \alpha} \sigma_{i j} \tau_{i j}\left\{t_{i j}^{n} \bar{f}_{i \alpha} f_{j \alpha}+\tilde{t}^{\Delta} a_{i j}\left[f_{i \uparrow} f_{j \downarrow}-(\uparrow \rightarrow \downarrow)\right]+\text { c.c. }\right\} \\
-\sum_{i \alpha} \bar{f}_{i \alpha} f_{i \alpha}, \\
S_{c}=-\sum_{i j} t_{i j}^{c} \tau_{i j}\left(b_{c i}^{*} b_{c j}+\text { c.c. }\right), \\
S_{s}=-\sum_{i j} t_{i j}^{s} \sigma_{i j}\left(z_{i}^{*} z_{j}+\text { c.c. }\right), \\
S_{\sigma \tau}=-K_{\sigma} \prod_{\square} \sigma_{i j}-K_{\tau} \prod_{\square} \tau_{i j}-K_{\sigma \tau} \prod_{\square} \sigma_{i j} \tau_{i j} .
\end{gathered}
$$

Here $b_{c}$ is a charge $e$ spinless boson and $z$ is a spin- $1 / 2$ chargeless boson. The $f$ field represents a spinless, neutral fermion (the spin index is just a label with no special significance). The $\sigma_{i j}$ and $\tau_{i j}$ are two independent $Z_{2}$ gauge fields. The physical electron $c_{i \alpha}=b_{c i} z_{i} f_{i \alpha}$. Clearly if the $\tau$ field is confining, the $f_{\alpha}$ and $b_{c}$ get confined to form a Fermionic holon-we then recover the action discussed earlier in this section. On the other hand, if the $\sigma$ field is confining, the Fermi statistics gets glued to the spinon $\left(z_{i}\right)$-the resulting theory is essentially that introduced in Ref. 7 in the context of cuprate physics and involves Bosonic holons and Fermionic spinons coupled to a $Z_{2}$ gauge field. If both gauge fields $\sigma$ and $\tau$ are deconfining, however, we have an exotic phase in which the fields $b_{c}, z, f$ are all liberated. This phase will also have two distinct vison excitations corresponding to the fluxes of the two $Z_{2}$ gauge fields. We may view this phase as a higher-level fractionalized phase as compared to the one discussed in Ref. 7 or that discussed earlier in this section. The connection between various fractionalized phases is shown in Fig. 1. We use symbols $b_{a}$ and $f_{a}$ to label bosons and fermions that carry quantum numbers $a=n, c, s, c s$ (neutral, charge, spin, charge, and spin) and show the existence of appropriate $Z_{2}$ vortices in each phase (for more details see Sec. V).

In the sections which follow, we will show how an effective action such as that of Eq. (32) can arise in the context of $p$-wave superconducting systems and systems which feature interplay between magnetism and superconductivity.

\section{FRACTIONALIZATION OF ELECTRON QUANTUM NUMBERS WITH $p$-WAVE PAIRING}

\section{A. Order parameters and symmetries}

Spin-triplet superconductors and their rich orderparameter structure offer the prospect of various exotic phases. Since they break both charge and spin symmetries, triplet superconductors exhibit features of both singlet superconductors and of spin models. In particular, we can envision the restoration of the the $\mathrm{U}_{C}(1)$ charge symmetry by quantum fluctuations, thereby resulting in a spin-triplet insulating state. Alternatively, the spin symmetry (we will make the simplifying assumption that the system has only an easyplane $\mathrm{U}_{S}(1)$ spin symmetry) can be restored, resulting in a spin-singlet superconducting state. Finally, both symmetries can be restored, leading to a singlet insulating state. We believe that the gapped, symmetry-restored states will not be very sensitive to the precise symmetry of the spin sector, so we believe that our results apply to systems with full SU(2) spin symmetry as well. In particular, when the symmetry is increased (while keeping the size of the representation fixed), fluctuations are enhanced, and a system is more likely to be in a disordered state. In order for these symmetries to be restored separately, it will be necessary, as we discuss below, for a type of topological ordering to occur. This topological ordering is essentially spin-charge separation of the charge $2 e$, spin-triplet Cooper pairs. Depending on the way in which the symmetries are restored, it is possible for further topological ordering to take place, in which case the quantum disordered states may support excitations with exotic quantum numbers. In such states, the spin and/or charge of the quasiparticles is screened by the Goldstone modes (which are themselves separated from each other by the higher-level topological ordering). As we describe in this paper, there are no fewer than nine phase which can result in this way.

To be concrete, let us consider the following $p$-wave superconducting state of electrons on a square lattice:

$$
\Delta_{\alpha \beta}=\Delta_{0} e^{i \varphi}\left(\cos \theta \sigma_{\alpha \beta}^{z}+i \sin \theta \delta_{\alpha \beta}\right) \sin k_{y} a .
$$

This is the most general unitary triplet state in two dimensions ${ }^{20}$ if we assume that there is only the $\mathrm{U}(1)$ spin symmetry of rotations about the $z$ axis, rather than the full SU(2). In Eq. (33), only $\Delta_{\uparrow \uparrow}$ and $\Delta_{\downarrow \downarrow}$ are nonzero. The lower symmetry could be the result of spin-orbit coupling. The symmetry-breaking pattern associated with this order parameter is: $\mathrm{U}_{C}(1) \times \mathrm{U}_{S}(1) \times D_{4} \rightarrow Z_{2} \times Z_{2} \times D_{2}$. The $\mathrm{U}_{C}(1)$ charge symmetry is broken to $Z_{2}$ by the condensation of a charge $2 e$ order parameter. The $\mathrm{U}_{S}(1)$ spin-rotational symmetry is completely broken. The square lattice point group, $D_{4}$, is broken to $D_{2}$ by the orbital symmetry of $\Delta$. Finally, there is an additional $Z_{2}$ since the order parameter is left invariant by $\varphi \rightarrow \varphi+\pi, \theta \rightarrow \theta+\pi$. As we discuss later, this can be understood as a $Z_{2}$ gauge symmetry. From $e^{i \varphi}$ and $e^{i \theta}$ we can construct the following $Z_{2}$-invariant order parameters whose presence or absence characterizes the phases which we consider. In the absence of the triplet $p$-wave supercon- 


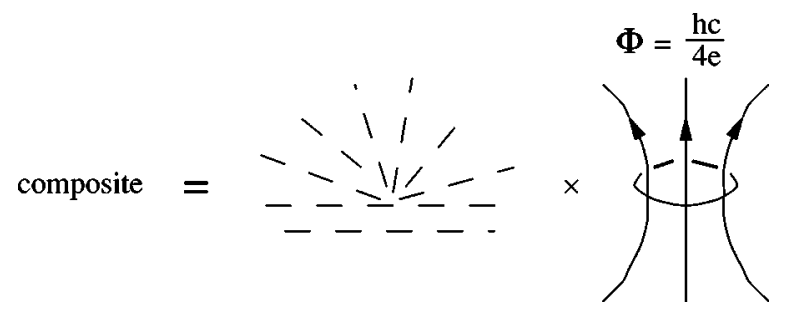

FIG. 2. $\pi$-disclination- $h c / 4 e$ vortex composite.

ducting order parameter (33), we can characterize states by the charge $-4 e$ order parameter,

$$
\Delta^{4 e}=\left(e^{i \varphi}\right)^{2},
$$

and the spin nematic order parameter,

$$
Q=\cos 2 \theta .
$$

These order parameters define the following quantumdisordered states of triplet $p$-wave superconductors.

- Charge-4e singlet superconductor: $\Delta^{4 e} \neq 0, Q=0$.

- Charge-4e nematic superconductor: $\Delta^{4 e} \neq 0, Q \neq 0$.

- Spin-nematic insulator: $\Delta^{4 e}=0, Q \neq 0$.

- Spin-singlet insulator: $\Delta^{4 e}=0, Q=0$.

\section{B. Topological defects}

The quantum-disordered and topologically ordered states which we will consider can be understood in terms of the condensation or suppression of various topological excitations. The most basic and fundamental topological excitation is a composite formed of a flux $h c / 4 e$ vortex together with a $\pi$ disclination. ${ }^{34-36}$

Along a circuit about such an excitation, both $\varphi$ and $\theta$ wind by $\pi$ so that any $Z_{2}$-invariant combination is singlevalued. If such an excitation is at the origin, and $r, \phi$ are polar coordinates in the plane, then the order parameter is of the form

$$
\Delta_{\alpha \beta}(r, \phi)=\Delta(r) e^{i \epsilon_{1} \phi / 2}\left(\cos \frac{\phi}{2} \sigma_{\alpha \beta}^{z}+i \epsilon_{2} \sin \frac{\phi}{2} \delta_{\alpha \beta}\right) \sin k_{y} a,
$$

where $\Delta(0)=0$ and $\Delta(\infty)=\Delta_{0}$. The flux is into or out of the plane, respectively, for $\epsilon_{1}= \pm 1$; the spins wind clockwise or counterclockwise, respectively, for $\epsilon_{2}= \pm 1$. It is instructive to write this as

$$
\begin{gathered}
\Delta_{\uparrow \uparrow}(r, \phi)=\Delta(r) e^{i \epsilon_{+} \phi} \sin k_{y} a, \\
\Delta_{\downarrow \downarrow}(r, \phi)=-\Delta(r) e^{i \epsilon_{-} \phi} \sin k_{y} a,
\end{gathered}
$$

where $\epsilon_{ \pm}=\left(\epsilon_{1} \pm \epsilon_{2}\right) / 2$. Hence, $\pi$-disclination-hc/4e vortex composites are vortices in $\Delta_{\uparrow \uparrow}$ or $\Delta_{\downarrow \downarrow}$ alone (see Fig. 2).

These excitations can be combined to form an $h c / 2 e$ vortex which is nontrivial in the charge sector but trivial in the spin sector,

$$
\Delta_{\alpha \beta}(r, \phi)=\Delta(r) e^{i \phi}\left(\cos \theta_{0} \sigma_{\alpha \beta}^{z}+i \sin \theta_{0} \delta_{\alpha \beta}\right) \sin k_{y} a
$$

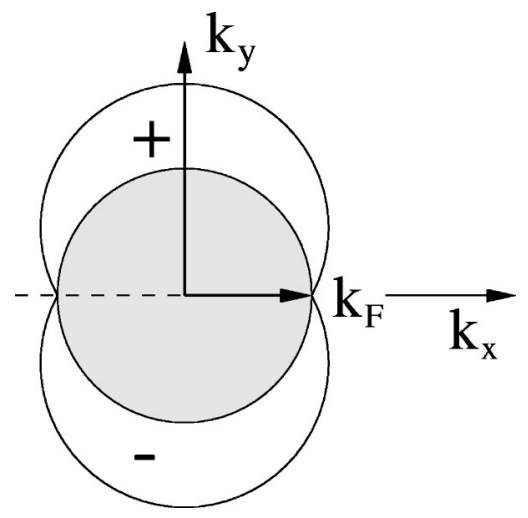

FIG. 3. Order parameter for a $\sin k_{y} p$-wave superconductor. Gapless excitations exist at $\vec{k}_{F}=\left( \pm k_{F}, 0\right)$.

with constant $\theta_{0}$. Alternatively, we can form merons, which are trivial in the charge sector but not the spin sector,

$$
\Delta_{\alpha \beta}(r, \phi)=\Delta(r) e^{i \varphi_{0}}\left(\cos \phi \sigma_{\alpha \beta}^{z}+i \sin \phi \delta_{\alpha \beta}\right) \sin k_{y} a
$$

with constant $\varphi_{0}$. Finally, there are various composites formed from the above. A composite formed by $n h c / 2 e$ vortices and $m$ merons takes the form

$$
\Delta_{\alpha \beta}(r \rightarrow \infty, \phi)=\Delta_{0} e^{i n \phi}\left(\cos m \phi \sigma_{\alpha \beta}^{z}+i \sin m \phi \delta_{\alpha \beta}\right) \sin k_{y} a .
$$

If flux $h c / 4 e$ vortex- $\pi$ disclination composites condense, then $\mathrm{U}_{C}(1)$ and $\mathrm{U}_{S}(1)$ are restored. The system will be in a singlet insulating state and all excitations will have conventional quantum numbers. If, on the other hand, $h c / 4 e$ vortex$\pi$ disclination composites are gapped and only complexes consisting of multiples of $h c / 4 e$ vortex- $\pi$ disclinations (e.g., $n$ hc/2e- $m$ meron composites) are condensed, then quantum number separation is possible. If complexes consisting of a multiple of four $h c / 4 e$ vortex- $\pi$ disclinations condense, then we will have the various versions of quantum number separation summarized in Figs. 3 and 4.

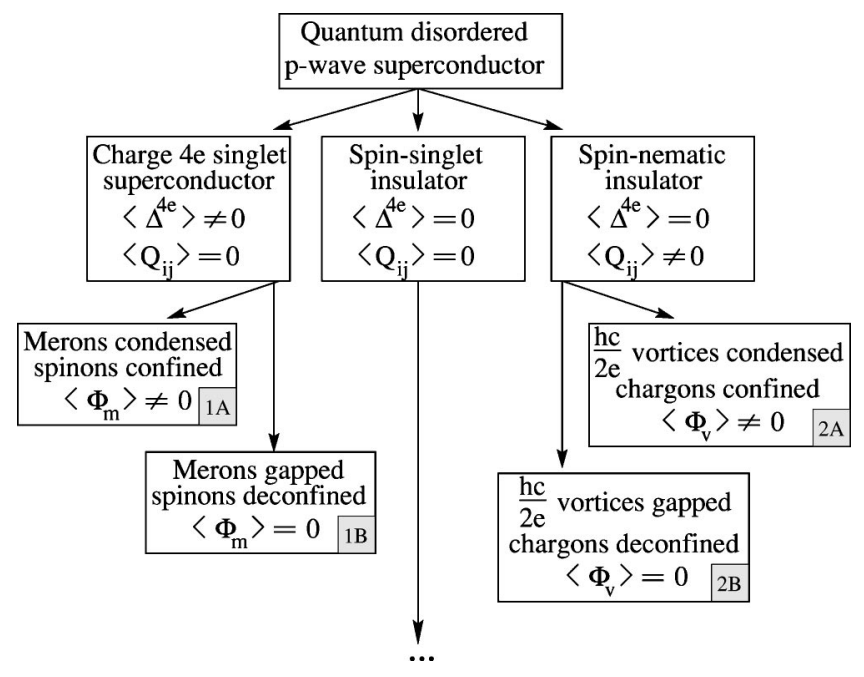

FIG. 4. Phases of quantum-disordered $p$-wave superconductor. 


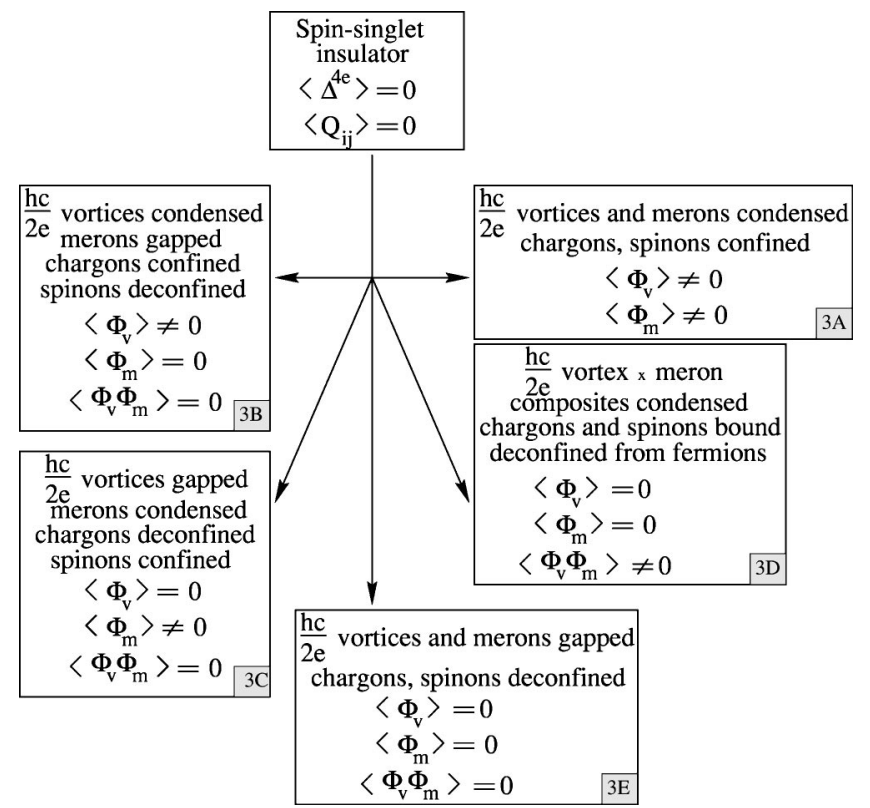

FIG. 5. Phases of quantum-disordered $p$-wave superconductor. Note that the phase in which $h c / 2 e$ vortices and merons are condensed may be described as having $h c / 4 e$-vortex- $\pi$ composites condensed.

\section{Quantum number separation}

The effective action of a $p$-wave superconductor may be written in the form

$$
S_{\mathrm{tot}}=S_{f}+S_{c}+S_{\sigma}
$$

where $S_{f}$ is the action for the Fermionic quasiparticles and their interactions with the Goldstone modes, and $S_{c}$ and $S_{\sigma}$ are the actions for the charge and spin Goldstone modes.

Depending on the topology of the Fermi surface, the lowenergy spectrum of a $p$-wave superconductor may include gapless Fermionic quasiparticles. Let us assume that the topology is such that the gap has nodes on the Fermi surface. Focusing on the nodes, as shown in Fig. 5. We linearize the action

$$
\begin{aligned}
S_{f}= & \int d^{2} x d \tau \chi^{\dagger}\left[\partial_{\tau}-A_{\tau}^{c} \tau^{z}-v_{F} \tau^{z} i \partial_{x}+v_{F} A_{x}^{c}-A_{\tau}^{\sigma} \sigma_{z}\right. \\
& +v_{F} A_{x}^{\sigma} \sigma_{z} \tau_{z}-v_{\Delta} \tau^{s} e^{i s \varphi}\left(\cos \theta \sigma_{\alpha \beta}^{x}+i \sin \theta \sigma_{\alpha \beta}^{y}\right) \\
& \left.\times\left(i \partial_{y}\right)\right] \chi .
\end{aligned}
$$

$s= \pm$ and $\chi$ has a particle-hole index, acted on by Pauli matrices $\vec{\tau}$; and a spin index, acted on by Pauli matrices $\vec{\sigma}$,

$$
\chi_{a \alpha}(\vec{k})=\left[\begin{array}{c}
\chi_{11} \\
\chi_{21} \\
\chi_{12} \\
\chi_{22}
\end{array}\right]=\left[\begin{array}{c}
c_{\vec{k}_{F}+\vec{k} \uparrow} \\
c_{-\vec{k}_{F}-\vec{k} \downarrow}^{\dagger} \\
c_{\vec{k}_{F}+\vec{k} \downarrow} \\
-c_{-\vec{k}_{F}-\vec{k} \uparrow}^{\dagger}
\end{array}\right]
$$

In action (42) we have included the electromagnetic field $A_{\mu}^{c}$ and spin vector potential $A_{\mu}^{\sigma}$ which couple to the conserved electric and $S_{z}$ currents.

When $h c / 4 e$ vortex- $\pi$ disclination composites are gapped, the $Z_{2}$ symmetry $\varphi \rightarrow \varphi+\pi, \theta \rightarrow \theta+\pi$ plays no role, and the other terms in Eq. (41) may be written in the form

$$
S_{c}=\frac{1}{2} \rho_{c} \int d^{2} x d \tau\left(\partial_{\mu} \varphi-A_{\mu}^{c}\right)^{2}
$$

and

$$
S_{\sigma}=\frac{1}{2} \rho_{\sigma} \int d^{2} x d \tau\left(\partial_{\mu} \theta-A_{\mu}^{\sigma}\right)^{2}
$$

The conserved electric and $S_{z}$ currents are given by

$$
j_{\mu}^{c, \sigma}=\frac{\delta S_{\mathrm{tot}}}{\delta A_{\mu}^{c, \sigma}} .
$$

Conservation of charge and the $z$ component of spin require

$$
\partial_{\mu} j_{\mu}^{c, \sigma}=0
$$

The interactions between the Goldstone fields and the quasiparticles are highly nonlinear in Eq. (42). This interaction can be made more tractable, following Ref. 28 , if we define new fermion fields $\psi$ :

$$
\chi=e^{i \varphi \tau^{z} / 2} e^{i \theta \sigma^{z} / 2} \psi
$$

With this change of variables, we have defined a neutral, spinless fermion $\psi$, which is governed by the action

$$
\begin{aligned}
S_{f}= & \int d^{2} x d \tau\left(\psi^{\dagger}\left[\partial_{\tau}-v_{F} \tau^{z} i \partial_{x}-v_{\Delta} \tau^{x} \sigma^{x}\left(i \partial_{y}\right)\right] \psi\right. \\
& +\frac{1}{2} \psi^{\dagger}\left[\tau^{z} \partial_{\tau} \varphi-2 A_{\tau}^{c} \tau^{z}-v_{F} \partial_{x} \varphi+2 v_{F} A_{x}^{c}\right] \psi \\
& \left.+\frac{1}{2} \psi^{\dagger}\left[\sigma^{z} \partial_{\tau} \theta-2 A_{\tau}^{\sigma} \tau^{z}-v_{F} \tau^{z} \sigma^{z} \partial_{x} \theta+2 v_{F} \tau^{z} \sigma^{z} A_{x}^{\sigma}\right] \psi\right) .
\end{aligned}
$$

The couplings between the Goldstone modes and the quasiparticles are now either trilinear or biquadratic,

$$
\begin{aligned}
S_{f}= & S_{f}^{0}+\frac{1}{2} \int d^{2} x d \tau\left[J_{0}^{c}\left(\partial_{\tau} \varphi-2 A_{\tau}^{c}\right)+J_{x}^{c}\left(\partial_{x} \varphi-2 A_{x}^{c}\right)\right. \\
& \left.+J_{0}^{\sigma}\left(\partial_{\tau} \theta-2 A_{\tau}^{\sigma}\right)+J_{x}^{\sigma}\left(\partial_{x} \theta-2 A_{x}^{\sigma}\right)\right]
\end{aligned}
$$

with

$$
\begin{gathered}
J_{0}^{c}=\psi^{\dagger} \tau^{z} \psi, \quad J_{x}^{c}=-v_{F} \psi^{\dagger} \psi \\
J_{0}^{\sigma}=\psi^{\dagger} \sigma^{z} \psi, \quad J_{x}^{\sigma}=-v_{F} \psi^{\dagger} \sigma^{z} \tau^{z} \psi
\end{gathered}
$$

The price that must be paid is that the change of variables (48) is not single valued about a topological defect. In par- 
ticular, the charge part, $\exp \left(i \varphi \tau^{z} / 2\right)$, is double valued under transport about a flux $h c / 2 e$ vortex since $\varphi$ winds by $2 \pi$, while the spin part, $\exp \left(i \theta \sigma^{z} / 2\right)$, is double valued under transport about a meron since $\theta$ winds by $2 \pi$.

As we will see below, $\psi$ s are weakly coupled quasiparticles in those quantum disordered phases in which flux $h c / 2 e$ vortices and merons are gapped.

\section{Defect condensation}

Defect condensation is now implemented with dual representations for the order parameters. ${ }^{28,13,29,37}$ In the dual description of the $X Y$ model,${ }^{37}$ the ordering field $\varphi$ is replaced by a gauge field which parametrizes the total current, together with a vortex field which accounts for the singularities of $\varphi$.

We use the conservation of charge to define the dual gauge field,

$$
\epsilon_{\mu \nu \lambda} \partial_{\nu} a_{\lambda}^{c}=J_{\mu}^{\text {tot } c}=\rho_{c}\left(\partial_{\mu} \varphi-A_{\mu}^{c}\right)+J_{\mu}^{c},
$$

with $J_{\mu}^{c}$ from Eq. (51), and introduce the vortex current,

$$
j_{\mu}^{v}=\frac{1}{2 \pi} \epsilon_{\mu \nu \lambda} \partial_{\nu} \partial_{\lambda} \varphi,
$$

which is not vanishing for a multivalued $\varphi$. With the last two equations we can relate the vortex current to the dual gauge field $a_{\mu}^{c}$ and quasiparticle current $J_{\mu}^{c}$,

$$
j_{\mu}^{v}=\epsilon_{\mu \nu \lambda} \partial_{\nu}\left[\rho_{c}^{-1} \epsilon_{\lambda \alpha \beta} \partial_{\alpha} a_{\beta}^{c}+A_{\lambda}^{c}-\rho_{c}^{-1} J_{\lambda}^{c}\right] .
$$

Now a dual action for the charged degrees of freedom is easily constructed by requiring that its equations of motion reproduce Eq. (54),

$$
\begin{aligned}
S_{\text {Dual }}^{c}= & S_{G L}\left(\Phi_{v}, a_{\mu}^{c}\right)+\int d \tau d^{2} x\left[\frac{1}{2 \rho_{c}}\left(f_{\mu \nu}^{c}\right)^{2}\right. \\
& \left.+a_{\mu}^{c} \epsilon_{\mu \nu \lambda} \partial_{\nu}\left(A_{\lambda}^{c}-\frac{1}{\rho_{c}} J_{\lambda}^{c}\right)\right],
\end{aligned}
$$

where

$$
S_{G L}\left[\Phi, a_{\mu}\right]=\int d \tau d^{2} x\left(\frac{\rho_{d}}{2}\left|\left(\partial_{\mu}-i a_{\mu}\right) \Phi\right|^{2}+V(\Phi)\right)
$$

and $f_{\mu \nu}^{c}=\partial_{\nu} a_{\mu}^{c}-\partial_{\mu} a_{\nu}^{c}$. The field $\Phi_{v}^{\dagger}$ may be thought of as a vortex creation field. The vortex current is given by

$$
j_{\mu}^{v}=\frac{\rho_{d}}{2}\left[\Phi_{v}^{\dagger}\left(\frac{1}{i} \partial_{\mu}-a_{\mu}^{c}\right) \Phi_{v}+\text { H.c. }\right] .
$$

An identical construction is now used for $\theta$ with $\rho_{c}$ replaced by $\rho_{\sigma}$ and $J_{\lambda}^{c}$ by $J_{\lambda}^{\sigma}$ :

$$
\begin{aligned}
S_{\text {Dual }}^{\sigma}= & S_{G L}\left(\Phi_{m}, a_{\mu}^{\sigma}\right)+\int d \tau d^{2} x\left[\frac{1}{2 \rho_{\sigma}}\left(f_{\mu \nu}^{\sigma}\right)^{2}\right. \\
& \left.+a_{\mu}^{\sigma} \epsilon_{\mu \nu \lambda} \partial_{\nu}\left(A_{\lambda}^{\sigma}-\frac{1}{\rho_{c}} J_{\lambda}^{\sigma}\right)\right],
\end{aligned}
$$

where $\Phi_{m}^{\dagger}$ is a meron creation operator. Analogous topological objects in the spin sector have been discussed in the context of quantum Hall systems ${ }^{38-40}$ and quantum antiferromagnets..$^{17,41-43}$

Actions (56) and (58) need to be supplemented by ChernSimons gauge fields which enforce the minus sign which is acquired when a $\psi$ encircles a flux $h c / 2 e$ vortex or a meron ${ }^{28,44}$ With these additions, we obtain the following dual action:

$$
\begin{aligned}
S_{\text {Dual }}= & S_{G L}\left(\Phi_{v}, a_{\mu}^{c}-a_{\mu}^{s 1}\right)+S_{G L}\left(\Phi_{m}, a_{\mu}^{\sigma}-a_{\mu}^{s 2}\right) \\
& +\int d \tau d^{2} x\left[\frac{1}{2 \rho_{c}}\left(f_{\mu \nu}^{c}\right)^{2}+a_{\mu}^{c} \epsilon_{\mu \nu \lambda} \partial_{\nu}\left(A_{\lambda}^{c}-\frac{1}{\rho_{c}} J_{\lambda}^{c}\right)\right. \\
& \left.+2 \alpha_{\mu}^{1} \epsilon_{\mu \nu \lambda} \partial_{\nu} a_{\lambda}^{s 1}+\alpha_{\mu}^{1} J_{\mu}^{c}\right]+\int d \tau d^{2} x\left[\frac{1}{2 \rho_{\sigma}}\left(f_{\mu \nu}^{\sigma}\right)^{2}\right. \\
& \left.+a_{\mu}^{\sigma} \epsilon_{\mu \nu \lambda} \partial_{\nu}\left(A_{\lambda}^{\sigma}-\frac{1}{\rho_{\sigma}} J_{\lambda}^{\sigma}\right)+2 \alpha_{\mu}^{2} \epsilon_{\mu \nu \lambda} \partial_{\nu} a_{\lambda}^{s 2}+\alpha_{\mu}^{2} J_{\mu}^{\sigma}\right],
\end{aligned}
$$

where $\alpha_{\mu}^{1,2}$ and $a_{\mu}^{s 1,2}$ are the gauge fields that perform the flux attachement and enforce the minus sign.

With this action in hand, we can now address the quantum disordered phases and quantum number separation. In essence, there are three quantum numbers: charge, spin, and electron number modulo 2 . These can separate in a variety of patterns.

- If $\left\langle\Phi_{v}\right\rangle \neq 0$, flux $h c / 2 e$ vortices condense. The Meissner effect associated with this condensate imposes

$$
a_{\mu}^{c}+a_{\mu}^{s 1}=0 .
$$

Recalling that $\epsilon_{\alpha \beta} \partial_{\alpha} a_{\beta}^{c}=J_{0}^{\text {totc }}(\alpha, \beta=x, y)$ is the charge density and $\epsilon_{\alpha \beta} \partial_{\alpha} a_{\beta}^{s 1}=J_{0}^{c}$ is the quasiparticle density, we conclude that in this phase charge is attached to the $\psi \mathrm{s}$.

- If $\left\langle\Phi_{m}\right\rangle \neq 0$, merons condense, and the Meissner effect associated with this condensate imposes

$$
a_{\mu}^{\sigma}+a_{\mu}^{s 2}=0 .
$$

As $\epsilon_{\alpha \beta} \partial_{\alpha} a_{\beta}^{\sigma}=J_{0}^{\text {tot } \sigma}$ is the local spin density and $\epsilon_{\alpha \beta} \partial_{\alpha} a_{\beta}^{s 2}=J_{0}^{\sigma}$ we find that spin is attached to the $\psi$ 's. All the fermions carry spin.

- If $\left\langle\Phi_{m}\right\rangle=0,\left\langle\Phi_{v}\right\rangle=0$, but $\left\langle\Phi_{v} \Phi_{m}\right\rangle \neq 0 h c / 2 e$ vortex, meron composites condense. The Meissner effect associated with this condensate imposes

$$
a_{\mu}^{c}+a_{\mu}^{\sigma}=0 .
$$

In other words, spin and charge are confined, but the fermion $\psi$ carries neither since $\psi$ does not acquire any phase upon encircling this composite object, as evinced by the fact that $\Phi_{v} \Phi_{m}$ is not coupled to statistical gauge fields.

- The condensation of other composites, such as $\Phi_{m}^{2}$ (i.e., skyrmions), $\Phi_{v}^{2}$, etc., does not cause the confinement of any quantum numbers.

\section{E. Exotic phases}

The order-parameter classification discussed after Eq. (35) is incomplete; those states can occur in several varieties, classified by the allowed quantum numbers. ${ }^{45}$ 
Charge-4e singlet superconductors, $\Delta^{4 e} \neq 0, Q=0$ :

(1A) If $\left\langle\Phi_{m}\right\rangle \neq 0$, then the Fermionic excitations carry spin $1 / 2$.

(1B) However, if $\left\langle\Phi_{m}\right\rangle=0$ but $\left\langle\left(\Phi_{m}\right)^{2}\right\rangle \neq 0$ then the $\psi$ 's are spinless. Note that the charge quantum number of the Fermionic excitation is not really well defined since $\mathrm{U}(1)$ is broken in the superconducting state; stated differently, the fermion can always exchange charge with the condensate.

Spin-triplet insulator, $\Delta^{4 e}=0: Q \neq 0$ :

(2A) If $\left\langle\Phi_{v}\right\rangle \neq 0$, the $\psi$ 's carry charge $e$.

(2B) If $\left\langle\Phi_{v}\right\rangle=0$ but $\left\langle\left(\Phi_{v}\right)^{2}\right\rangle \neq 0$, then the $\psi$ 's are neutral. As in the previous case, the spin quantum number of the $\psi$ 's is not well defined.

Spin-singlet insulator, $\Delta^{4 e}=0: Q=0$ :

(3A) If $\left\langle\Phi_{v}\right\rangle \neq 0,\left\langle\Phi_{m}\right\rangle \neq 0$, then the $\psi$ 's carry spin $1 / 2$ and charge $e$ : CSF phase.

(3B) If $\left\langle\Phi_{m}\right\rangle=0$ but $\left\langle\left(\Phi_{m}\right)^{2}\right\rangle \neq 0$ while $\left\langle\Phi_{v}\right\rangle \neq 0$, then the $\psi$ 's are charge $e$, spinless Fermionic excitations: CFSB phase.

(3C) If $\left\langle\Phi_{v}\right\rangle=0$ but $\left\langle\left(\Phi_{v}\right)^{2}\right\rangle \neq 0$ while $\left\langle\Phi_{m}\right\rangle \neq 0$, then the $\psi$ 's are neutral, spin-1/2 Fermionic excitations: CBSF phase.

(3D) If $\left\langle\Phi_{v} \Phi_{m}\right\rangle \neq 0$, then the $\psi$ 's are neutral, spinless Fermionic excitations, but spin and charge are confined into a bosonic spin-1/2, charge $e$ excitation: CSBNF phase.

(3E) Finally, if $\left\langle\Phi_{v}\right\rangle=0$ but $\left\langle\left(\Phi_{v}\right)^{2}\right\rangle \neq 0$ and $\left\langle\Phi_{m}\right\rangle=0$ but $\left\langle\left(\Phi_{m}\right)^{2}\right\rangle \neq 0$, then the $\psi$ 's are neutral, spinless Fermionic excitations. Bosonic charge $e$ excitations, $e^{i \varphi / 2}$, and Bosonic spin-1/2 excitations, $e^{i \theta / 2}$ are also liberated: CBSBNF phase.

To summarize, we have the following phases with exotic quantum numbers:

-A charge-4e singlet superconductor with spinless Fermionic excitations.

-A spin-triplet insulator with neutral Fermionic excitations.

-Spin-singlet insulators with (i) charge $e$ spinless fermions and spin-1/2 neutral bosons; (ii) spin-1/2 neutral fermions and spinless charge $e$ bosons; (iii) neutral spinless fermions, Bosonic charge $e$ spinless excitations, and Bosonic spin-1/2 neutral excitations; or (iv) neutral spinless Fermionic excitations and Bosonic charge $e$ spin- $1 / 2$ excitations.

These result are summarized in the following diagrams that describe various phases that can result from quantum disordering a $p$-wave superconductor.

The scenario proposed in this section for quantum number separation in $p$-wave superconductors may apply to other systems, provided that they acquire nontrivial topological order in the spin and charge sectors, or in the language of this section when they have sufficiently strong quantum fluctuations of spin and charge degrees of freedom simultaneously. In Appendix $\mathrm{B}$ we show that quantum disordered $d$-wave superconductor with easy-plane antiferromagnetic fluctuations may be treated in the same way as we treated $p$-wave superconductors in this section.

\section{V. $Z_{2} \times Z_{2}$ LATTICE GAUGE THEORY}

In this section, we derive a $Z_{2} \times Z_{2}$ gauge theory representation of a model which gives rise to local $p$-wave superconducting fluctuations. In addition to the superconducting state, we find the exotic phases discussed in the previous section. These have a simple description as the various deconfining phases of the $Z_{2} \times Z_{2}$ gauge theory. Readers who are uninterested in the technical details of our derivation may skip directly to Eqs. (105), (106), and the subsequent discussion.

\section{A. General formalism}

We consider the following Hamiltonian that describes the equal spin pairing state of a $p$-wave superconductor:

$$
H=H_{t}+H_{u}+H_{v}+H_{\Delta}
$$

with

$$
\begin{gathered}
H_{t}=-t \sum_{r r^{\prime}, \alpha} c_{r \alpha^{\prime}}^{\dagger} c_{r^{\prime} \alpha}+\text { H.c. }, \\
H_{u}=u \sum_{r}\left(N_{r}-N_{0}\right)^{2}, \\
H_{v}=v \sum_{r}\left(M_{r}\right)^{2}, \\
H_{\Delta}=\sum_{r r^{\prime}}\left[\Delta_{r r^{\prime}}^{\uparrow \uparrow} c_{r \uparrow} c_{r^{\prime} \uparrow}+\Delta_{r r^{\prime}}^{\downarrow \downarrow} c_{r \downarrow} c_{r^{\prime} \downarrow}\right]+\text { H.c. },
\end{gathered}
$$

where $\Delta_{r r^{\prime}}^{\uparrow \uparrow}$, and $\Delta_{r r^{\prime}}^{\downarrow \downarrow}$, represent the order-parameter fields for the Cooper pairs with spin up-up and down-down pairs, respectively. Here $\alpha=\uparrow, \downarrow$ is the spin index. The term proportional to $u$ represents the on-site Coulomb repulsion. $N_{r}$ is the total number operator of electrons at the site $r, N_{0}$ is the average electron number per site. $M_{r}$ is the $z$ component of the total spin operator. At equilibrium, $\left|\Delta^{\uparrow \uparrow}\right|=\left|\Delta^{\downarrow \downarrow}\right|$. Note that there are two independent phases associated with $\Delta^{\uparrow \uparrow}$ and $\Delta^{\downarrow \downarrow}$. We can rewrite $H_{\Delta}$ as

$$
H_{\Delta}=\Delta \sum_{r r^{\prime}} a_{r r^{\prime}}\left[e^{i \varphi_{r \uparrow}} c_{r \uparrow} c_{r^{\prime} \uparrow}+e^{i \varphi_{r \downarrow}} c_{r \downarrow} c_{r^{\prime} \downarrow}\right]+\text { H.c., }
$$

where $\Delta=\left|\Delta^{\uparrow \uparrow}\right|=\left|\Delta^{\downarrow \downarrow}\right|$ and $a_{r r}$, is the form factor that gives rise to the particular $p$-wave symmetry.

The fields $\varphi_{r \uparrow}$ and $\varphi_{r \downarrow}$ are canonically conjugate to the Cooper pair number operators of up-up and down-down Cooper pairs, $n_{r \uparrow}$ and $n_{r \downarrow}$ :

$$
\left[\varphi_{r \uparrow}, n_{r^{\prime} \uparrow}\right]=i \delta_{r r^{\prime}}, \quad\left[\varphi_{r \downarrow}, n_{r^{\prime} \downarrow}\right]=i \delta_{r r^{\prime}} .
$$

The conserved charge densities for the electrons with spin $\uparrow$ and $\downarrow$ are given by

$$
\begin{aligned}
& N_{r \uparrow}=2 n_{r \uparrow}+\rho_{r \uparrow}, \\
& N_{r \downarrow}=2 n_{r \downarrow}+\rho_{r \downarrow},
\end{aligned}
$$

where $\rho_{r \alpha}=c_{r \alpha}^{\dagger} c_{r \alpha}$ is the quasiparticle number, which is not equal to the electron number. It is useful to remind the readers that the Hamiltonian (64) does not conserve the quasiparticle number, since it contains terms that annihilate a pair of 
them and create a Cooper pair. Only the total number of electrons of a given spin, given by Eq. (67), is conserved. This may be formulated as a conservation of the total charge and $z$ component of the total spin,

$$
N_{r}=N_{r \uparrow}+N_{r \downarrow}, \quad M_{r}=N_{r \uparrow}-N_{r \downarrow} .
$$

Let us define boson operators $b_{r \alpha}$ which carry charge $e$ and $\operatorname{spin} \alpha=\uparrow, \downarrow$ :

$$
b_{r \alpha}^{\dagger}=t_{r}^{\alpha} e^{i \varphi_{r \alpha} / 2}=e^{i \phi_{r \alpha}},
$$

where $t_{r}^{\alpha}= \pm 1$ are Ising variables and $\phi_{r \alpha}$ are defined in the interval zero to $2 \pi$. Note that the squares of $b_{r \uparrow}^{\dagger}$ and $b_{r \downarrow}^{\dagger}$ create the spin up-up and down-down Cooper pairs via the following relation:

$$
\left(b_{r \alpha}^{\dagger}\right)^{2}=e^{i \varphi_{r \alpha}} .
$$

One can also see that the canonical conjugates of $\phi_{r \alpha}$ are the total densities of electrons with spin $\uparrow$ and $\downarrow$. They satisfy the following commutation relations:

$$
\left[\phi_{r \alpha}, N_{r^{\prime} \alpha}\right]=i \delta_{r r^{\prime}} .
$$

Similarly, the following commutation relations are also satisfied:

$$
\left[\phi_{r c}, N_{r^{\prime}}\right]=i \delta_{r r^{\prime}}, \quad\left[\phi_{r s}, M_{r^{\prime}}\right]=i \delta_{r r^{\prime}},
$$

where $\phi_{r c}=\left(\phi_{r \uparrow}+\phi_{r \downarrow}\right) / 2$ and $\phi_{r s}=\left(\phi_{r \uparrow}-\phi_{r \downarrow}\right) / 2$. At this stage, it is useful to define the fermion operators, $f_{r \alpha}^{\dagger}$, as follows:

$$
c_{r \alpha}^{\dagger}=b_{r \alpha}^{\dagger} f_{r \alpha}^{\dagger} .
$$

Note that $f_{r \alpha}^{\dagger}$ creates spinless neutral fermions due to the fact that $b_{r \alpha}^{\dagger}$ carries both the charge and spin of the electrons.

It is also useful to define $\varphi_{r c}$ and $\varphi_{r s}$ as follows:

$$
e^{i \varphi_{r \uparrow}}=e^{i \varphi_{r c}} e^{i \varphi_{r s}}, \quad e^{i \varphi_{r \downarrow}}=e^{i \varphi_{r c}} e^{-i \varphi_{r s}} .
$$

Note that there is a $Z_{2}$ symmetry associated with these definitions of phase variables; $\varphi_{r c} \rightarrow \varphi_{r c}+\pi$ and $\varphi_{r s} \rightarrow \varphi_{r s}+\pi$ do not change $e^{i \varphi_{r \uparrow}}$ and $e^{i \varphi_{r \downarrow}}$. Now we define boson operators $b_{r}^{\dagger}$ and $z_{r}^{\dagger}$ as

$$
b_{r}^{\dagger}=t_{r} e^{i \varphi_{r c} / 2}=e^{i \phi_{r c}}, \quad z_{r}^{\dagger}=s_{r} e^{i \varphi_{r s} / 2}=e^{i \phi_{r s}} .
$$

Here $t_{r}= \pm 1$ and $s_{r}= \pm 1$ are Ising variables. Note that these operators satisfy the following identities:

$$
\left(b_{r}^{\dagger}\right)^{2}=e^{i \varphi_{r c}}, \quad\left(z_{r}^{\dagger}\right)^{2}=e^{i \varphi_{r s} .}
$$

Note also that $b_{r \uparrow}^{\dagger}$ and $b_{r \downarrow}^{\dagger}$ can be rewritten as

$$
b_{r \uparrow}^{\dagger}=b_{r}^{\dagger} z_{r}^{\dagger}, \quad b_{r \downarrow}^{\dagger}=b_{r}^{\dagger} z_{r} .
$$

Now the total Hamiltonian can be written as

$$
H=H_{t}+H_{u}+H_{v}+H_{\Delta}
$$

with

$$
\begin{gathered}
H_{t}=-t \sum_{r r^{\prime}}\left(b_{r}^{\dagger} b_{r^{\prime}} z_{r}^{\dagger} z_{r^{\prime}} f_{r \uparrow}^{\dagger} f_{r^{\prime} \uparrow}+b_{r}^{\dagger} b_{r^{\prime}} z_{r^{\prime}} z_{r^{\prime}}^{\dagger} f_{r^{\prime} \downarrow}^{\dagger} f_{r^{\prime} \downarrow}\right)+\text { H.c., } \\
H_{\Delta}=\Delta \sum_{r r^{\prime}} a_{r r^{\prime}}\left(b_{r}^{\dagger} b_{r^{\prime}} z_{r}^{\dagger} z_{r^{\prime}} f_{r \uparrow} f_{r^{\prime} \uparrow}+b_{r}^{\dagger} b_{r^{\prime}} z_{r} z_{r^{\prime}}^{\dagger} f_{r \downarrow} f_{r^{\prime} \downarrow}\right)+\text { H.c. }
\end{gathered}
$$

The Hamiltonian is invariant under the following local transformations:

(i) $\quad Z_{2 \tau}: b_{r} \rightarrow-b_{r} ; f_{r \alpha} \rightarrow-f_{r \alpha}$;

(ii) $Z_{2 \sigma}: z_{r} \rightarrow-z_{r} ; f_{r \alpha} \rightarrow-f_{r \alpha}$;

(iii) $Z_{2 \tilde{\sigma}}: z_{r} \rightarrow-z_{r} ; b_{r \alpha} \rightarrow-b_{r \alpha}$.

Only two of these transformations are independent, any one of them can be represented as a product of the other two. Together they form $Z_{2} \times Z_{2}$ gauge symmetry, that has three $Z_{2}$ subgroups as reflected in three possible transformations above. These subgroups are distinct, but not independent. $Z_{2} \times Z_{2}$ local gauge symmetry is a consequence of the redundancy in the enlarged Hilbert space of $f_{r \alpha}, b_{r}$, and $z_{r}$. There is a further redundancy in our description in terms of $b_{r}$ and $z_{r}$ because $b_{r} \rightarrow i b_{r}, z_{r} \rightarrow-i z_{r}$ also leaves all physical quantities invariant. This identification allows for the existence of flux $\pi$-disclination- $h c / 4 e$ vortex composites which we discussed in Sec. IV B. As before, we assume that these topological defects are gapped so that we can safely ignore this identification and take $\phi_{r c}$ and $\phi_{r s}$ as defined from $[0,2 \pi)$.

In order to get the correct Hilbert space of the electrons, we have to impose two constraints at each site.

$$
\begin{aligned}
& N_{r}+\rho_{r \uparrow}+\rho_{r \downarrow}=\text { even number, } \\
& M_{r}+\rho_{r \uparrow}-\rho_{r \downarrow}=\text { even number. }
\end{aligned}
$$

These can be written as

$$
(-1)^{N_{r}+\rho_{r \uparrow}+\rho_{r \downarrow}}=1, \quad(-1)^{M_{r}+\rho_{r \uparrow}-\rho_{r \downarrow}}=1 .
$$

The constraints can be implemented in the path integral representation of the partition function using the following projection operators:

$$
\mathcal{P}_{c}=\prod_{r} \mathcal{P}_{r c}, \quad \mathcal{P}_{s}=\prod_{r} \mathcal{P}_{r s},
$$

with

$$
\begin{aligned}
\mathcal{P}_{r c} & =\frac{1}{2}\left[1+(-1)^{\left.N_{r}+\rho_{r \uparrow}+\rho_{r \downarrow}\right]}\right. \\
& =\frac{1}{2} \sum_{\sigma_{r}= \pm 1} e^{i(\pi / 2)\left(1-\tau_{r}\right)\left(N_{r}+\rho_{r \uparrow}+\rho_{r \downarrow}\right)}, \\
\mathcal{P}_{r s} & =\frac{1}{2}\left[1+(-1)^{\left.M_{r}+\rho_{r \uparrow}-\rho_{r \downarrow}\right]}\right. \\
& =\frac{1}{2} \sum_{\tau_{r}= \pm 1} e^{i(\pi / 2)\left(1-\sigma_{r}\right)\left(M_{r}+\rho_{r \uparrow}-\rho_{r \downarrow}\right)} .
\end{aligned}
$$


Using the projection operators, the partition function can be written as

$$
Z=\operatorname{Tr}\left[e^{-\beta H} \mathcal{P}_{c} \mathcal{P}_{s}\right]
$$

A Euclidean path-integral representation can be obtained by splitting the exponential into $M$ number of time slices,

$$
Z=\operatorname{Tr}\left[\left(e^{-\epsilon H} \mathcal{P}_{c} \mathcal{P}_{s}\right)^{M}\right],
$$

where $\epsilon=\beta / M$. Now the partition function can be written as

$$
\begin{aligned}
Z= & \int \prod_{i \alpha} d \bar{f}_{i \alpha} d f_{i \alpha} d \phi_{i c} d \phi_{i s} \\
& \times \sum_{N_{i}=-\infty}^{\infty} \sum_{M_{i}=-\infty}^{\infty} \sum_{\sigma_{i}= \pm 1} \sum_{\tau_{i}= \pm 1} e^{-S} .
\end{aligned}
$$

Here $i=(r, \tau)$ runs over the $2+1$-dimensional space-time lattice with $\tau=1,2, \ldots, M$ time slices. The action $S$ has the following form:

$$
S=S_{\tau}^{f}+S_{\tau}^{\phi_{c}}+S_{\tau}^{\phi_{s}}+\epsilon \sum_{\tau=1}^{M} H\left(N_{\tau}, M_{\tau}, \phi_{\tau c}, \phi_{\tau s}, \bar{f}_{\tau \alpha}, f_{\tau \alpha}\right)
$$

with

$$
\begin{aligned}
& S_{\tau}^{f}=\sum_{r, \tau=1}^{M} \sum_{\alpha}\left[\bar{f}_{\tau \alpha}\left(\sigma_{\tau+1} \tau_{\tau+1} f_{\tau+1, \alpha}-f_{\tau \alpha}\right)\right], \\
& S_{\tau}^{\phi_{c}}=\sum_{r, \tau=1}^{M} N_{\tau}\left(\phi_{\tau c}-\phi_{\tau-1, c}+\frac{\pi}{2}\left(1-\tau_{\tau}\right)\right), \\
& S_{\tau}^{\phi_{s}}=\sum_{r, \tau=1}^{M} M_{\tau}\left(\phi_{\tau s}-\phi_{\tau-1, s}+\frac{\pi}{2}\left(1-\sigma_{\tau}\right)\right) .
\end{aligned}
$$

Here the spatial index $r$ is suppressed for clarity. The Ising variables $\sigma_{\tau}$ and $\tau_{\tau}$ are defined on the links connecting adjacent time slices and can be regarded as the time component of the Ising gauge fields.

The sum of $H_{t}$ and $H_{\Delta}$ can be decoupled using the Hubbard-Stratanovich fields $\chi_{r r^{\prime}}$ and $\eta_{r r^{\prime}}$,

$$
e^{-\epsilon\left(H_{t}+H_{\Delta}\right)}=\int \prod_{r r^{\prime}} \prod_{\tau} d \chi_{r r^{\prime}} d \chi_{r r^{\prime}}^{*} d \eta_{r r^{\prime}} d \eta_{r r^{\prime}}^{*} e^{-S_{t, \Delta}} .
$$

Using the expressions for $H_{t}$ and $H_{\Delta}$,

$$
\begin{gathered}
H_{t}=-t \sum_{r r^{\prime}, \alpha}\left(b_{r \alpha}^{\dagger} b_{r^{\prime} \alpha} f_{r \alpha}^{\dagger} f_{r^{\prime} \alpha}+\text { H.c. }\right), \\
H_{\Delta}=\Delta \sum_{r r^{\prime}, \alpha} a_{r r^{\prime}}\left(b_{r \alpha}^{\dagger} b_{r^{\prime} \alpha} f_{r \alpha} f_{r^{\prime} \alpha}+\text { H.c. }\right),
\end{gathered}
$$

we have

$$
\begin{aligned}
S_{t, \Delta}= & \frac{1}{4} \epsilon \sum_{r r^{\prime}, \alpha}\left\{\left[2\left|\chi_{r r^{\prime}}\right|^{2}-\chi_{r r^{\prime}}\left(b_{r \alpha}^{*} b_{r^{\prime} \alpha}+t \bar{f}_{r \alpha} f_{r^{\prime} \alpha}\right.\right.\right. \\
& \left.\left.+a_{r r^{\prime}} \Delta f_{r \alpha} f_{r^{\prime} \alpha}\right)\right]+\left[2\left|\eta_{r r^{\prime}}\right|^{2}-\eta_{r r^{\prime}}\left(b_{r \alpha}^{*} b_{r^{\prime} \alpha}\right.\right. \\
& \left.\left.\left.-t \bar{f}_{r \alpha} f_{r^{\prime} \alpha}-a_{r r^{\prime}} \Delta f_{r \alpha} f_{r^{\prime} \alpha}\right)\right]+ \text { c.c. }\right\} .
\end{aligned}
$$

Rearranging terms, we get

$$
\begin{aligned}
S_{t, \Delta}= & \frac{1}{4} \epsilon \sum_{r r^{\prime}, \alpha}\left[2\left|\chi_{r r^{\prime}}\right|^{2}+2\left|\eta_{r r^{\prime}}\right|^{2}-\left(\chi_{r r^{\prime}}+\eta_{r r^{\prime}}\right) b_{r \alpha}^{*} b_{r^{\prime} \alpha}\right. \\
& -t\left(\chi_{r r^{\prime}}-\eta_{r r^{\prime}}\right) \bar{f}_{r \alpha} f_{r^{\prime} \alpha}-a_{r r^{\prime}} \Delta\left(\chi_{r r^{\prime}}-\eta_{r r^{\prime}}\right) f_{r \alpha} f_{r^{\prime} \alpha} \\
& + \text { c.c. }] .
\end{aligned}
$$

Rewriting this in terms of $b_{r}$ and $z_{r}$, we get

$$
\begin{aligned}
S_{t, \Delta}= & \frac{1}{4} \epsilon \sum_{r r^{\prime}}\left\{\sum _ { \alpha } \left[2\left|\chi_{r r^{\prime}}\right|^{2}+2\left|\eta_{r r^{\prime}}\right|^{2}\right.\right. \\
& \left.-t\left(\chi_{r r^{\prime}}-\eta_{r r^{\prime}}\right) \bar{f}_{r \alpha} f_{r^{\prime} \alpha}-a_{r r^{\prime}} \Delta\left(\chi_{r r^{\prime}}-\eta_{r r^{\prime}}\right) f_{r \alpha} f_{r^{\prime} \alpha}\right] \\
& \left.-\left(\chi_{r r^{\prime}}+\eta_{r r^{\prime}}\right)\left(b_{r}^{\dagger} b_{r^{\prime}} z_{r}^{\dagger} z_{r^{\prime}}+b_{r}^{\dagger} b_{r^{\prime}} z_{r} z_{r^{\prime}}^{\dagger}\right)+\text { c.c. }\right\} .
\end{aligned}
$$

In order to decouple $b_{r}$ from $z_{r}$, another HubbardStratanovich transformation is necessary. Using similar procedures, the term

$$
-\frac{1}{4} \epsilon \sum_{r r^{\prime}}\left[\left(\chi_{r r^{\prime}}+\eta_{r r^{\prime}}\right)\left(b_{r}^{\dagger} b_{r^{\prime}} z_{r^{\prime}}^{\dagger} z_{r^{\prime}}+b_{r}^{\dagger} b_{r^{\prime}} z_{r} z_{r^{\prime}}^{\dagger}\right)+\text { c.c. }\right]
$$

can be decoupled as

$$
\begin{aligned}
& -\frac{1}{16} \epsilon \sum_{r r^{\prime}}\left(\chi_{r r^{\prime}}+\eta_{r r^{\prime}}\right)\left[2\left|\lambda_{r r^{\prime}}\right|^{2}+2\left|\xi_{r r^{\prime}}\right|^{2}+2\left|p_{r r^{\prime}}\right|^{2}\right. \\
& \quad+2\left|q_{r r^{\prime}}\right|^{2}-\left(\lambda_{r r^{\prime}}+\xi_{r r^{\prime}}\right) z_{r}^{\dagger} z_{r^{\prime}}-\left(p_{r r^{\prime}}+q_{r r^{\prime}}\right) z_{r} z_{r^{\prime}}^{\dagger} \\
& \left.\quad-\left(p_{r r^{\prime}}-q_{r r^{\prime}}+\lambda_{r r}-\xi_{r r^{\prime}}\right) b_{r}^{\dagger} b_{r^{\prime}}+\text { c.c. }\right],
\end{aligned}
$$

We now make a saddle-point approximation and keep the Ising fluctuations around this saddle point. The natural choices are

$$
\begin{gathered}
\chi_{r r^{\prime}}-\eta_{r r^{\prime}}=\sigma_{r r^{\prime}} \tau_{r r^{\prime}} \chi_{f}, \\
\left(\chi_{r r^{\prime}}+\eta_{r r^{\prime}}\right)\left(\lambda_{r r^{\prime}}-\xi_{r r^{\prime}}+p_{r r^{\prime}}-q_{r r^{\prime}}\right)=\tau_{r r^{\prime}} \chi_{c}, \\
\left(\chi_{r r^{\prime}}+\eta_{r r^{\prime}}\right)\left(\lambda_{r r^{\prime}}+\xi_{r r^{\prime}}+p_{r r^{\prime}}^{*}+q_{r r^{\prime}}^{*}\right)=\sigma_{r r^{\prime}} \chi_{s},
\end{gathered}
$$

where $\sigma_{r r^{\prime}}= \pm 1$ and $\tau_{r r^{\prime}}= \pm 1$ are Ising fluctuations. We drop all of the constant terms and define the following variables:

$$
t_{f}=\frac{1}{4} t \chi_{f}, \quad t_{c}=\frac{1}{16} \chi_{c}, \quad t_{s}=\frac{1}{16} \chi_{s}, \quad t_{\Delta}=\frac{1}{4} \Delta \chi_{f}
$$

to obtain 


$$
\begin{aligned}
S_{t, \Delta}^{\mathrm{eff}}= & -\epsilon \sum_{r r^{\prime}} \sum_{\alpha}\left[\sigma_{r r^{\prime}} \tau_{r r^{\prime}}\left(t_{f} \bar{f}_{r \alpha} f_{r^{\prime} \alpha}+t_{\Delta} a_{r r^{\prime}} f_{r \alpha} f_{r^{\prime} \alpha}\right)\right. \\
& \left.+t_{c} \tau_{r r^{\prime}} b_{r}^{\dagger} b_{r^{\prime}}+t_{s} \sigma_{r r^{\prime}} z_{r}^{\dagger} z_{r^{\prime}}+\text { c.c. }\right] .
\end{aligned}
$$

Combining all the results, the approximate full partition function can be written as

$$
\begin{aligned}
\widetilde{Z}= & \int \prod_{i \alpha} d \bar{f}_{i \alpha} d f_{i \alpha} d \phi_{i c} d \phi_{i s} \\
& \times \sum_{N_{i}=-\infty} \sum_{M_{i}=-\infty} \prod_{\langle i j\rangle} \sum_{\sigma_{i j}= \pm 1} \sum_{\tau_{i j}= \pm 1} e^{-S},
\end{aligned}
$$

where $\sigma_{i j}$ and $\tau_{i j}$ are $Z_{2}$ gauge fields living on the nearest neighbor links of the space-time lattice. The total action $S$ is given by

$$
S=S_{\tau}^{f}+S_{\tau}^{\phi_{c}}+S_{\tau}^{\phi_{s}}+S_{\Delta}+S_{0}+S_{u}+S_{v}
$$

with

$$
\begin{gathered}
S_{\tau}^{f}=-i \sum_{i, j=i+\hat{\tau}} \sum_{\alpha}\left[\bar{f}_{i \alpha}\left(\sigma_{i j} \tau_{i j} f_{j \alpha}-f_{i \alpha}\right)\right], \\
S_{\tau}^{\phi_{c}=-i} \sum_{i, j=i-\hat{\tau}} N_{i}\left[\phi_{i c}-\phi_{j c}+\frac{\pi}{2}\left(1-\tau_{i j}\right)\right], \\
S_{\tau}^{\phi_{s}=-i} \sum_{i, j=i-\hat{\tau}} M_{i}\left[\phi_{i s}-\phi_{j s}+\frac{\pi}{2}\left(1-\sigma_{i j}\right)\right], \\
S_{\Delta}=\epsilon \sum_{i, j=i+\hat{x}} t_{\Delta} \sigma_{i j} \tau_{i j}\left(a_{i j} f_{i \alpha} f_{j \alpha}+\text { c.c. }\right), \\
S_{0}=-\epsilon \sum_{i, j=i+\hat{x}} \sum_{\alpha}\left[t_{f} \sigma_{i j} \tau_{i j} \bar{f}_{i \alpha} f_{j \alpha}\right. \\
\left.+t_{c} \tau_{i j} b_{i}^{*} b_{j}+t_{s} \sigma_{i j} z_{i}^{*} z_{j}+\text { c.c. }\right], \\
S_{u}=\epsilon u \sum_{i}\left(N_{i}-N_{0}\right)^{2}, \\
S_{v}=\epsilon v \sum_{i}\left(M_{i}\right)^{2},
\end{gathered}
$$

where $\hat{\tau}$ and $\hat{x}$ represent the time and spatial links. $a_{i j}$ $=a_{r r^{\prime}}$ on the spatial links and zero otherwise.

Using the Poisson resummation formula, one can show that

$$
\begin{gathered}
\sum_{N_{i}} e^{-\left(S_{u}+S_{\tau}^{\phi_{c}}\right)}=e^{\Sigma_{i, j=i-\hat{\tau}}(1 / 2 \epsilon u) \tau_{i j} \cos \left(\phi_{i c}-\phi_{j c}\right)-S_{B}^{\sigma},} \\
\sum_{M_{i}} e^{-\left(S_{v}+S_{\tau}^{\phi_{s}}\right)}=e^{\Sigma_{i, j=i-\hat{\tau}}(1 / 2 \epsilon v) \sigma_{i j} \cos \left(\phi_{i s}-\phi_{j s}\right)}
\end{gathered}
$$

with

$$
S_{B}^{\tau}=-i N_{0} \sum_{i, j=i-\hat{\tau}}\left(2 \pi l_{i j}^{\tau}-\frac{\pi}{2}\left(1-\tau_{i j}\right)\right) .
$$

Here $l_{i j}^{\tau}$ is defined as

$$
l_{i j}^{\tau}=\operatorname{Int}\left[\frac{\Phi_{i j}^{c}}{2 \pi}+\frac{1}{2}\right]
$$

with $\Phi_{i j}^{c}=\phi_{i c}-\phi_{j c}+(\pi / 2)\left(1-\tau_{i j}\right)$ is the gauge invariant phase difference across the temporal link. Int denotes the integer part. One can see that the Berry phase term for $\sigma_{i j}$ is absent. This is due to the fact that we have equal amplitudes for up-up and down-down pairing in the equal spin pairing state, analogous to particle-hole symmetry in the charge sector. by

Gathering these terms, the final form of the action is given

$$
S=S_{f}+S_{c}+S_{s}+S_{B}^{\sigma}+S_{g}
$$

with

$$
\begin{gathered}
S_{f}=-\sum_{i j, \alpha} \sigma_{i j} \tau_{i j}\left[t_{i j}^{f} \bar{f}_{i \alpha} f_{j \alpha}+\tilde{t}^{\Delta} a_{i j} f_{i \alpha} f_{j \alpha}+\text { c.c. }\right]-\sum_{i \alpha} \bar{f}_{i \alpha} f_{i \alpha}, \\
S_{c}=-\sum_{i j} t_{i j}^{c} \tau_{i j}\left(b_{i}^{*} b_{j}+\text { c.c. }\right) \\
S_{s}=-\sum_{i j} t_{i j}^{s} \sigma_{i j}\left(z_{i} z_{j}+\text { c.c. }\right) .
\end{gathered}
$$

Here $t_{i j}^{c}$ is $\epsilon t_{c}$ on the spatial link and $1 / 4 \epsilon u$ on the temporal link. Similarly $t_{i j}^{s}$ is $\epsilon t_{s}$ on the spatial link and $1 / 4 \epsilon v$ on the temporal link. $t_{i j}^{f}=\epsilon t_{f}$ on the spatial link and $t_{i j}^{f}=-1$ on the temporal link. And $\tilde{t}^{\Delta}=\epsilon t_{\Delta}$. The last term of Eq. (105) corresponds to the Maxwell terms for the $Z_{2}$ gauge fields, that we assume are generated after we integrate out excitations at high energies,

$$
S_{g}=-K_{1} \sum_{\square} \prod_{\square} \sigma_{i j}-K_{2} \sum_{\square} \prod_{\square} \tau_{i j}-K_{3} \sum_{\square} \prod_{\square} \sigma_{i j} \tau_{i j} .
$$

These are the simplest terms providing dynamics of the gauge fields that are consistent with the gauge symmetries,

$$
\begin{aligned}
& Z_{2 \tau}: \quad b_{i} \rightarrow t_{i} b_{i} ; \quad f_{i \alpha} \rightarrow t_{i} f_{i \alpha} ; \quad \tau_{i j} \rightarrow t_{i} t_{j} \tau_{i j}, \\
& Z_{2 \sigma}: \quad z_{i} \rightarrow s_{i} z_{i} ; \quad f_{i \alpha} \rightarrow s_{i j} f_{i \alpha} ; \quad \sigma_{i j} \rightarrow s_{i} s_{j} \sigma_{i j},
\end{aligned}
$$

where $t_{i}$ and $s_{i}$ are \pm 1 . In the future we will call any particle that transforms under the first and the second transformations of Eq. (108) as having $Z_{2 \tau}$ and $Z_{2 \sigma}$ charges, respectively.

\section{B. Spin singlet insulating phases}

Before discussing possible spin singlet insulating phases of the combined action (105)-(107) it is useful to review properties of a pure $Z_{2} \times Z_{2}$ gauge theory (107). Under dual- 
ity transformation defined in Refs. 7,46 and 47 this model becomes a generalized Ashkin-Teller model, ${ }^{48}$

$$
S_{A T}=-K_{d 1} \sum_{\langle i j\rangle} v_{i} v_{j}-K_{d 2} \sum_{\langle i j\rangle} u_{i} u_{j}-K_{d 3} \sum_{\langle i j\rangle} u_{i} v_{i} u_{j} v_{j}
$$

Here $u_{i}$ and $v_{i}$ are Ising variables defined on the dual lattice in $d=2+1$. We can identify five possible phases of Eq. (109):

fully ordered phase $\langle u\rangle \neq 0,\langle v\rangle \neq 0,\langle u v\rangle \neq 0 ;$

(ii) partially ordered phase $\langle u\rangle \neq 0,\langle v\rangle=0,\langle u v\rangle=0$;

(iii) partially ordered phase $\langle u\rangle=0,\langle v\rangle \neq 0,\langle u v\rangle=0$;

(iv) partially ordered phase $\langle u\rangle=0,\langle v\rangle=0,\langle u v\rangle \neq 0$;

(v) disordered phase $\langle u\rangle=0,\langle v\rangle=0,\langle u v\rangle=0$.

As pointed out in Ref. 7 the Ising variables of Eq. (109) correspond to the $Z_{2}$ vortices of the original gauge model. They describe gauge field configurations with plaquette products equal to -1 , i.e., plaquettes pierced by $Z_{2}$ fluxes. Following Ref. 7 we call such $Z_{2}$ vortices "visons." In fact we have three kinds of visons: $\sigma$ visons that describe $Z_{2}$ vortices of $\sigma, \tau$ visons that describe $Z_{2}$ vortices of $\tau$, and $[\sigma \tau]$ visons that describe a composite of $\sigma$ and $\tau Z_{2}$ vortices. The three are not independent, any one of them can be thought of as a composite object of the other two. However, we should treat all of them on equal footing since they represent distinct topological objects. The appearance of the long range order in the Ashkin-Teller model corresponds to the condensation of visons in the original gauge model and describes transition to the confining phase. From these arguments it follows that there are five distinct phases of the pure gauge model in Eq. (107): one fully confining phase, three partially confining phases, and one fully deconfining phase, that correspond to the fully ordered, three partially ordered, and one fully disordered phases of the Ashkin-Teller model.

(i) Fully confining phase. $\sigma$ and $\tau$ visons are condensed simultaneously. This also implies condensation of $[\sigma \tau]$ visons.

(ii) Partially confining phase. $\tau$ visons are condensed and $\sigma$ and $[\sigma \tau]$ visons are gapped.

(iii) Partially confining phase. $\sigma$ visons are condensed and $\tau$ and $[\sigma \tau]$ visons are gapped.

(iv) Partially confining phase. $[\sigma \tau]$ visons are condensed and $\sigma$ and $\tau$ visons are gapped.

(v) Deconfining phase. All visons are gapped.

Condensation of visons has dramatic effects on the motion of spinons, holons, and neutral fermions in the model (105)-(107). We find drastically different excitation spectra depending on what vortices are condensed. The reason for this is a geometrical phase factor of $\pi$ that particles with $Z_{2}$ charges acquire when they circle around an appropriate $Z_{2}$ vortex. For example, spinons and neutral fermions get a geometrical phase factor of $\pi$ when they are transported around a $\sigma$ vison, and holons and neutral fermions get a minus sign when they circle around a $\tau$ vison. This means that when visons are present in the ground state, the coherent motion of the corresponding particles is highly frustrated and they may not be considered as elementary excitations. Only the particles that are neutral with respect to the appropriate $Z_{2}$ symmetry may propagate freely in a phase with condensed visons. And the particles that carry such $Z_{2}$ charges will have to bind into neutral pairs. This is the essence of the confinement argument discussed in Refs. 46 and 7.

When we apply the geometrical phase-confinement argument to the spin singlet insulating states we find the same phases as discussed in Sec. IV E.

- In a phase of type (i) all kinds of $Z_{2}$ vortices are condensed. Therefore particles that carry any $Z_{2}$ charges will be bound. This is a fully confining phase where only fully neutral composites are allowed. Holons, spinons, and neutral fermions are confined (phase CSF).

- In a phase of type (ii) we have a condensate of $\tau$ visons. As a result particles that carry $Z_{2 \tau}$ charges are confined, but particles that carry $Z_{2 \sigma}$ charges are liberated. Spinons are free, and holons are bound to the neutral fermions (phase CFSB).

- In a phase of type (iii), that has a condensate of $\sigma$ visons, we have a confinement of particles with $Z_{2 \sigma}$ charges and deconfinement of particles with $Z_{2 \tau}$ charges. Holons are free, and spinons are bound to the neutral fermions (phase CBSF).

- In a phase of type (iv) we do not have individual $\sigma$ and $\tau$ visons in the ground states, but only their composites, $[\sigma \tau]$ visons. The geometrical phase argument becomes somewhat subtle when we consider $[\sigma \tau]$ visons. Particles that carry either one of $Z_{2 \tau}$ or $Z_{2 \sigma}$ charges will get a $\pi$ phase shift when they circle around such a vortex. However, particles that carry both charges acquire no phase. So, in a $D$-type phase particles that carry one of the $Z_{2 \tau}$ or $Z_{2 \sigma}$ charges are confined, but particles that carry both charges are deconfined. Holons and spinons are bound, and neutral fermions are free (phase CSBNF).

- Finally, in a phase of type (v) we have no condensed visons, which means that all the particles are liberated. Holons, spinons, and neutral fermions are deconfined (phase CBSBNF).

\section{Broken-symmetry phases}

In this section we show using $Z_{2} \times Z_{2}$ theory that even states with the long-range order in the model (105)-(107), i.e., $p$-wave superconductors, spin singlet superconductors, nematic insulators, and nematic superconductors may differ in their topological ordering and carry the remnants of the spin-charge separation that appears so dramatically in the insulating phase.

We begin by reviewing the case of a $p$-wave superconductor.

- The simplest $p$-wave superconductor that may be deduced from the model (105)-(107) is when holons and spinons condense simultaneously, so the system acquires finite expectation values of $b$ and $z$. The geometrical phase argu- 
ment when applied to this system tells us that an isolated $h c / 2 e$ vortex or a meron are no longer well defined excitations, since they acquire a phase shift of $\pi$ when circling around a holon or a spinon, respectively. However, if we bind an $h c / 2 e$ vortex with a $\tau$ vison we find that this composite can propagate freely. The geometrical phases acquired by the two upon encircling a holon add up to 0 or $2 \pi$. Equivalently, a meron, when bound to a $\sigma$ vison, becomes a well defined excitation in the presence of spinon condensate.

- Another possible phase of a $p$-wave superconductor is when we condense holon pairs and spinons, i.e., $b^{2}$ and $z$. In this phase merons are still bound to $\sigma$ visons, however, $h c / 2 e$ vortices and $\tau$ visons are now deconfined. The original holons are reduced to Ising variables, which we can call $b$ isons, following Ref. 7. They carry the leftover of the charge symmetry, that was broken from $\mathrm{U}(1)$ to $Z_{2}$, and are well defined excitations in this phase.

- Analogously to the previous case we can consider a situation with condensed $b$ and $z^{2}$. This phase will have bound $h c / 2 e$ vortices and $\tau$ visons and liberated merons and $\sigma$ visons. Spinons become Ising variables, $z$ isons, that carry the residual $Z_{2}$ spin quantum numbers.

- A different type of a $p$-wave superconductor occurs when holon pairs and holon-spinon composites condense simultaneously, i.e., $b^{2}$ and $b z$ acquire expectation values (this also fixes the expectation value for $z^{2}$ ). In such a phase spinons and holons are reduced to a single Ising variable, since knowing $b$ automatically gives $z$. This $b z$ ison carries the residual spin-charge quantum number of the system. A stable topological object in this phase may be constructed by taking any two of the set $(h c / 2 e$ vortex, meron, $\tau$ vison, $\sigma$ vison).

- Finally, we may have a phase with condensed holon and spinon pairs, $b^{2}$ and $z^{2}$. This gives us separate $b$ isons, $z$ isons, $h c / 2 e$ vortices, merons, $\tau$ visons, and $\sigma$ visons.

The last four phases are the triplet analogs of the exotic SC* phase discussed in Ref. 7 in the case of singlet superconductors. We now consider the case of a spin-singlet superconductor.

- The simplest kind of a spin singlet superconductor occurs when we condense simultaneously holons $b$ and $\sigma$ visons. The former ensures confinement of $h c / 2 e$ vortices and $\tau$ visons, whereas the latter gives rise to binding of neutral fermions to spinons.

- Another possibility is to have a condensate of holon pairs $b^{2}$ and $\sigma$ visons. This liberates $h c / 2 e$ vortices and $\tau$ visons, produces $b$ isons that carry charge $Z_{2}$ number, and leaves neutral fermions bound to spinons.

- Another option is to have a condensate of bosons $b$ with gapped $\sigma$ visons. This means bound $h c / 2 e$ vortices and $\tau$ visons, and liberated neutral fermions and spinons.

- The most intriguing phase in this series is obtained when we condense holon pairs $b^{2}$ and holon $\sigma$ vison composites.
Excitations in this phase will be any pair from the set ( $h c / 2 e$ vortex, $\tau$ vison, $\psi, z$ ) and $b$ isons.

- Finally we can have a condensate of $b^{2}$ and gapped $\sigma$ visons. This gives unconfined $h c / 2 e$ vortices, $\tau$ visons, neutral fermions, spinons, and $b$ isons.

Of the five phases above, four of the last ones may be considered as $\mathrm{SC}^{*}$ phases.

The construction given above for $p$-wave superconducting states and spin singlet superconducting states may be generalized to the case of spin-nematic insulators and nematic superconductors. In those cases, just as in the two discussed above, we find five possible states. One of these is a traditional version, whereas the other four are of the unconventional $*$ variety that may be thought of as containing traces of quantum number separation.

The reader may be worried that we do not find $h c / 4 e$ vortices $\pi$ disclinations in our discussion of various phases of $p$-wave superconductors. As in the previous sections we assumed that these excitations have been gapped out [see discussion after Eq. (79)].

\section{DISTINGUISHING DIFFERENT FRACTIONALIZED PHASES}

In previous sections, we have seen how various fractionalized phases can arise in the context of Kondo lattice models and systems with a tendency towards $p$-wave superconductivity or superconductivity coexisting with magnetism. These phases can be described in the language of vortex and skyrmion condensation or in terms of a $Z_{2} \times Z_{2}$ gauge theory. However, one might wonder if these results are an artifact of these formalisms. In particular, one can ask how these phases can be distinguished-both as a matter of principle and as a practical experimental issue - from each other and from unfractionalized phases. As Wen ${ }^{11}$ and, more recently, Senthil and Fisher $^{4}$ have emphasized recently, their "topological order" -i.e., the sensitivity of the ground state to changes of the topology of the system-provides one means of distinguishing fractionalized phases.

This characterization of fractionalized phases is crucial because other heuristic definitions of fractionalized phases can fail. To see why this is so, consider the intuitively appealing statement that a fractionalized phase is distinguished from a conventional phase by asking for the lowest energy excitation with, for instance, spin $1 / 2$. In the conventional case, this would be an electron which also carries an electric charge $e$. In the fractionalized phases of the kind discussed above, one might expect that the corresponding excitation is a spinon which is charge neutral. However, this test for fractionalization fails if there is an attractive interaction between the holons and spinons which binds them into an electron at low energies. This could, in principle, happen without going through a phase transition. (Unlike in an unfractionalized phase, holons and spinons would still exist as unbound excitations, but at higher energies.) Then, the lowest energy excitation with spin $1 / 2$ is an electron (as opposed to a spinon) though the system is adiabatically connected to a fractionalized phase (see Refs. 13, 28, and 29 for a discussion of this 
effect). Furthermore, other tests such as the vanishing of the quasiparticle residue at some point of the Brillouin zone also fail in this situation. Hence we turn to the characterization in terms of the topological properties of the system.

Topologically ordered systems are partially characterized by their ground-state degeneracy on the annulus, the torus, or higher-genus manifolds, over and above any degeneracy which may be due to broken symmetry. Consider the CBSF phase. It has a twofold degenerate ground state on the annulus. The two ground states correspond to periodic and antiperiodic boundary conditions for holons and spinons as they encircle the center of the annulus. In either case, electrons themselves have periodic boundary conditions, as they must. In an unfractionalized phase, spinons and holons are confined within an electron so the two states are identical; the excitations which could distinguish them are not part of the spectrum. By the same reasoning, the CFSB and CSBNF phases also have two degenerate ground states on the annulus. By extension, all of these states have ground-state degeneracy $4^{g}$ on a genus $g$ surface. On the other hand, CBSBNF has four degenerate ground states. We can independently choose periodic or antiperiodic boundary conditions for the charge and spin bosons. The boundary conditions for the neutral fermions are then determined by the requirement that electrons must have periodic boundary conditions. On a genus $g$ surface, it has degeneracy $16^{g}$.

These degeneracies can be interpreted in terms of the vison spectra of the fractionalized states. The two ground states of CBSF on an annulus correspond to the presence or absence of a $\tau$ vison (i.e., a $v$ ) in the center of the annulus; the two ground states of CFSB correspond to the presence or absence of a $\sigma$ vison (a $\left.v^{\prime}\right)$; the two ground states of CSBNF correspond to the presence or absence of a $\sigma \tau$ vison in the center of the annulus. The four ground states of CBSBNF correspond to the presence or absence of $\sigma$ and $\tau$ visons in the center of the annulus. The interpretation of these ground states in terms of visons forms the basis for an experimental probe of topological order proposed by Senthil and Fisher. ${ }^{19}$ We will return to this issue later but let us, in the meantime, continue to pursue the question of the distinction in principle between different fractionalized phases.

Different states at the same level of fractionalization have the same ground-state degeneracy; CBSF, CFSB, and CSBNF all have two degenerate ground states on the annulus. In order to distinguish them, we must consider their quantum number spectra. CSBNF does not have spin-charge separation, i.e., it is not possible to isolate a charge- 0 , spin$1 / 2$ excitation at finite-energy cost. Furthermore it is possible to isolate a neutral Fermionic excitation. Both of these stand in contrast to CBSF and CFSB which exhibit spin-charge separation but do not support neutral Fermionic excitations. Hence, we conclude that CSBNF is distinct from the other two states despite having the same ground state degeneracy.

One might be tempted to conclude that CBSF and CFSB are distinct because the lowest-energy charged excitation is a boson in one phase and a fermion in another phase. However, if a holon in CBSF forms a bound state with a $\tau$ vison, the resulting bound state will be Fermionic; similarly, if a spinon in CBSF forms a bound state with a $\tau$ vison, the resulting bound state will be Bosonic. Hence, as a result of the seemingly innocuous formation of bound states, the CBSF and CFSB states appear to metamorphose into each other. Thus one is instead tempted to conclude that the CBSF and CFSB phases can be adiabatically connected to each other.

This contention is supported by considering the singlet superconducting state which results if holons condense in CBSF or if holon- $\sigma$ vison composites condense in CFSB (see Fig. 1). It is easy to see that the superconducting states in either case are conventional and are smoothly connected to a BCS state. The superconducting state can be disordered by vortex condensation. This will yield a fractionalized state (with a twofold degenerate ground state on an annulus) if vortex pairs condense but individual vortices are uncondensed. Since the result could be either CBSF or CFSB, this appears to support the possibility that there is no phase boundary between these phases in the part of the phase diagram near the singlet superconducting phase.

However, there is a logically possible alternative, namely that an operator which is irrelevant in the superconducting phase and at the critical point becomes relevant at the fixed points characterizing the fractionalized phases. In that case, the actual nature of the resulting fractionalized phase depends on short distance physics - the value of the coupling which is formally irrelevant in the superconductor-and is not uniquely dictated by knowing that there is proliferation of $h c / e$ and with $h c / 2 e$ vortices remaining gapped.

Despite this caveat, a scenario in which CBSF and CFSB are smoothly connected to each other in the vicinity of their transition to the superconducting state is appealing and plausible. This does not necessarily mean that CBSF and CFSB are not distinct phases. Their relationship could be similar to that between a liquid and a gas, which are separated by a first-order phase transition line which terminates at a critical point, beyond which a liquid and a gas can be adiabatically connected without crossing a phase-transition line. In Appendix $\mathrm{C}$, we show that precisely such a scenario does occur in simpler (though somewhat different) $Z_{2} \times Z_{2}$ gauge theory models. Thus we tentatively suggest that the first-order phase transition between the CBSF and CFSB phases terminates at a critical point. Beyond this critical point, there is no distinction between these phases, and it is in this region of the phase diagram that there is a phase transition to the superconducting phase.

\section{FLUX-TRAPPING EXPERIMENTS}

Let us now consider the practical issue of how we can identify whether a given system in an unknown phase is fractionalized or not and, if it is fractionalized, then what its fractionalization pattern is. To proceed, note first that the CBSF phase contains in it the seed of superconductivity. As argued in Ref. 7, condensing the charged boson provides a natural nonpairing route to superconductivity (of a conventional kind). Similarly, the CFSB phase contains in it the seed of magnetism-simply condensing the spinon leads to a conventional state with some kind of magnetic long-range order. However, it is possible to imagine a transition between 
the CFSB phase and a superconductor which occurs when a composite formed by a holon and a $\sigma$ vison condenses. Similarly, it is possible to imagine a transition between the CBSF phase and a magnetic phase which occurs when a composite formed by a holon and a $\sigma$ vison condenses.

The feature of most interest to the following discussion is simply that a direct phase transition should be possible between the CBSF and CFSB phases and a conventional superconductor. Upon going through such a phase transition, the visons of these phases acquire $h c / 2 e$ units of electromagnetic flux to become the $h c / 2 e$ vortices of the superconductor. This may be exploited to devise a sensitive test for the topological order in the CBSF phase, as argued in Refs. 19 and 4.

The test proceeds as follows. Consider an annular sample of a material which is in a conventional superconducting phase and let us suppose that we can tune the sample parameters adiabatically so that the sample makes transitions between the superconducting phase and the CBSF and CFSB phases. Suppose that $h c / 2 e$ units of electromagnetic flux are trapped in the annulus when the system is in its superconducting phase. There must also be a vison trapped in the annulus so that the holon condensate can have periodic boundary conditions (without which it would cost infinite energy): the antiperiodicity caused by the flux $h c / 2 e$ is cancelled by the antiperiodicity due to the vison. If the system is taken into the CBSF phase, the flux escapes since there is no holon condensate trapping it, but a vison will remain since it will cost energy (the vison gap) to unwind the antiperiodic boundary conditions of the (neutral) spinons. If the system is returned to the superconducting state, then it must generate flux $\pm h c / 2 e$ so that the holon condensate can again have periodic boundary conditions. The same analysis holds if we take the system into the CFSB phase except that we have to replace "holon" in the above description by "holon-vison composite." On the other hand, if the system undergoes a transition to an unfractionalized phase, then the vison can escape since there are no deconfined spinons or holons whose boundary conditions would be affected by its escape.

Of course, this experiment would simply be confirming the result which we arrived at in the previous section: that the CBSF and CFSB phases can be adiabatically continued into each other, particularly in the neighborhood of a conventional singlet superconducting phase.

Let us now consider a more complicated flux-trapping experiment in which, as an intermediate step, we take the system through the higher-level fractionalized phase, CBSBNF (see Fig. 1). This phase has two distinct vison excitations. One of these visons can be envisioned as a descendent of the $\tau$ vison of the CBSF phase; we will refer to this as $v$. The other can be envisioned as a descendent of the $\sigma$ vison of the CFSB phase or as a by-product of the further fractionalization of the fermionic spinon of CBSF; we will refer to this as $v^{\prime}$. A direct transition from CBSBNF to the CBSF phase occurs when the visons $v^{\prime}$ condense while that from CBSBNF to CFSB occurs when the visons $v$ condense. The presence of two distinct visons in the CBSBNF phase distinguishes it from the CBSF and CFSB phases-indeed it will have a ground-state degeneracy of 16 on a torus.
Now consider a conventional BCS superconductor. This is obtained from CBSF by condensing the holon. The fluxtrapping experiment performed by moving between the superconductor and CBSF gives a positive result. Now consider a modification of the experiment so that we start in the superconducting phase, move first to CBSF, then to CBSBNF, then back into CBSF before finally going back into the superconductor. This again gives a positive result since $v$ is trapped in the annulus and it can never escape. Upon making the transition between the CBSF and CBSBNF phases, a $v^{\prime}$ will be generated with probability $1 / 2$ since the ground state of CBSF with one $v$ will make a transition to either of the corresponding ground states of CBSBNF with equal probability. However, this $v^{\prime}$ will escape upon the transition from CBSBNF back to CBSF. Now consider a further modification in which we go all the way from the superconductor to the CFSB phase through the CBSF and CBSBNF phases and then return by the same route to the superconductor. The result of this experiment will be negative half of the time. This is because in going from CBSBNF to CFSB, the vison $v$ condenses. Thus $v$ which was trapped in the hole until the phase CBSBNF was reached can escape on moving into the CFSB phase. In going from CFSB back to CBSBNF, a $v$ is generated with probability $1 / 2$-the two ground states are obtained with equal proability. This $v$, if it is generated, will lead to the generation of flux $h c / 2 e$ in the superconducting state.

Hence, there appears to be a difference between the CBSF and CFSB which can be detected in this experiment. It appears that these phases cannot be continuously connectedsince the probability of a negative result for the flux-trapping experiment of the previous paragraph must jump from 0 to $1 / 2$-at least in the vicinity of the CBSBNF phase. This can be understood in the following terms. In the CBSBNF phase, there are two distinct types of visons, $v$ and $v^{\prime}$. If one or the other condensed, a transition occurs to CFSB or CBSF. The remaining vison in CBSF "remembers" that it is a $v$ vison. Meanwhile the vison in CFSB remembers that it is a $v^{\prime}$ vison. However, if we take the system far from CBSBNF so that a bound state can form between a $v$ and a holon and also between a $v$ and a spinon, then $v$ now looks like a $v^{\prime}$ and the distinction between the two phases is blurred. Combining this reasoning with that of the previous section, we propose the phase diagram of Fig. 6 .

\section{DISCUSSION}

When electrons interact strongly, a number of interesting phenomena are known to occur, including unconventional superconductivity and magnetism. As we have seen in this paper, many of the physical settings which give rise to these phenomena also have the potential to exhibit electron fractionalization. Different theoretical approaches, adapted to these specific systems, suggest seemingly different fractionalized phases. It is natural to ask if these phases are truly different and, if so, what their organizing principle is.

In this paper, we have pursued the idea ${ }^{3,4}$ that a crisp and coherent way of understanding quantum number fractionalization is provided by the concept of topological order intro- 

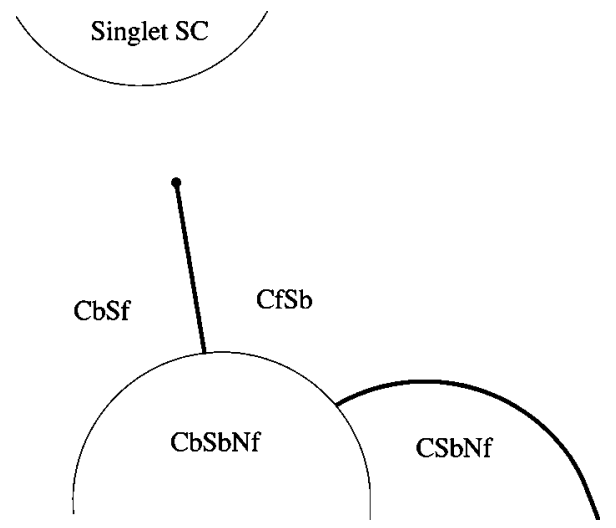

FIG. 6. A schematic phase diagram indicating how the CBSF, CFSB, CSBNF, CBSBNF, and conventional singlet superconducting phases might fit together. The thick lines are first-order phase transitions and the thin lines are second-order phase transitions.

duced in the context of fractional quantum Hall liquids, ${ }^{49}$ anyon superfluids, ${ }^{50}$ chiral spin states, ${ }^{51}$ and short-range resonating valence bond spin states. ${ }^{30}$ We presented two approaches for understanding such topological order. The first one relies on the recently developed $Z_{2}$ gauge theory of spincharge separation, originally suggested for the high- $T_{c}$ cuprates, and generalizes it to a $Z_{2} \times Z_{2}$ theory to include possible fractionalization of spin and charge quantum numbers. Some of the interesting fractionalized phases are: CBSF (Bosonic holons and Fermionic spinons), CFSB (Fermionic holons and Bosonic spinons), CSBNF (bound Bosonic holons and spinons and neutral fermions), and CBSBNF (Bosonic holons and spinons and neutral fermions). Any one of these phases can be further characterized by possible broken symmetries with conventional order parameters. Each of the fractionalized phases corresponds to a different deconfining phase of the pure $Z_{2} \times Z_{2}$ gauge theory and will have appropriate topological $Z_{2}$ vortices, visons, as finite-energy excitations.

An alternative picture of fractionalization which is also presented in this paper uses the language of quantum disordered superconductors and magnets. When topological ordering-defined by the suppression of certain defectsoccurs, the Goldstone modes associated with various broken symmetries can screen the corresponding quantum numbers of the Fermionic quasiparticles. In this way, these quasiparticles can be bleached of some or all of their quantum numbers. This may be implemented mathematically with U(1) particle-vortex duality in both the charge and spin sectors. We arrive at essentially the same picture as that of the $Z_{2}$ $\times Z_{2}$ gauge theory. In those insulating phases in which $h c / 2 e$ vortices are condensed, charge is bound to the Fermionic quasiparticles. When $h c / 2 e$ vortices are gapped and $h c / e$ vortices are condensed, charge carrying holons can propagate separately from the electrically neutral Fermionic quasiparticles. In the spin sector, we can consider either meron or skyrmion (which carry twice the topological charge of merons) condensation, with gapped merons in the latter case. In the former case, spin is confined to the Fermionic quasiparticles, and in the latter case spinons will exist as independent objects, deconfined from the Fermionic quasiparticles.
We have also discussed the possibility of quantum disordered phases in which the condensed topological objects are the $h c / 2 e$ vortex-meron composites, but not $h c / 2 e$ vortices or merons separately. Such phases have spinons and holons bound together but deconfined from the neutral Fermionic quasiparticles.

An important issue discussed in this paper is whether one can distinguish the phases obtained by quantum disordering the spin and charge sectors of the system, for example, the phases CFSB and CBSF of the quantum disordered $p$-wave superconductor. The simplest choice seems to be the identification of the spin excitation as a Fermionic or Bosonic particle. This, however, is not a reliable tool. In the $Z_{2} \times Z_{2}$ gauge theory formulation, both spinons and holons carry $Z_{2}$ charges, so a bound state of a $Z_{2}$ vortices with either one of them (this can also be thought of as attaching Wilson loops to the particles) will change its statistics from Fermionic to Bosonic or vice versa. ${ }^{17,42,43,52}$ In the deconfining phase such vortices are gapped. However, if a bound state between a $Z_{2}$ charge carrying particle and a $Z_{2}$ vortex forms, this bound state may have a lower energy than the original particle. This means that in both CFSB and CBSF phases the lowest energy spin- or charge-carrying excitations can exist as either bosons or fermions. The subtleties discussed above lead us to consider flux-trapping experiments of the type discussed in Sec. VII. Combining all of these considerations, we outlined one scenario in which CBSF and CFSB phases can be separated by a first-order transition which terminates at a critical point. On the other hand, one can go from CBSF to CFSB through CBSBNF phase by two continuous transitions. Thus, if this scenario is correct, the relation between CBSF and CFSB is somewhat similar to that between liquid and gas phases. We, however, defer offering any definitive conclusion.

Spin charge separation in one-dimensional systems is fundamentally different from its two-dimensional counterpart, since it does not involve topological order. Another nontrivial realization of electron number fractionalization which is analogous to that presented here can occur in multicomponent quantum Hall systems and was discussed in Refs. 40 and 53.

Another avenue for further research is the investigation of quantum-disordered states of triplet superconductors with more complicated spin structures appearing in some of the superfluid phases of ${ }^{3} \mathrm{He}$. We expect that these will share some features of noncollinear spin-density waves. ${ }^{54}$ Further exotic phases are likely to occur upon quantum-disordering states with multiple order parameters. We have considered one of the simplest cases of this-antiferromagnetism and superconductivity - but there are more complicated possibilities, involving incommensurate charge and/or spin order.

In addition to the phases CBSF and CFSB that have appeared in the literature before, we proposed the possibility of two additional quantum number separated phases in these systems: phase CSBNF in which the excitations are a spin$1 / 2$, charge $e$ boson, a neutral spinless fermion and a vison and phase CBSBNF with a charge $e$ spinless boson, a neutral spin $1 / 2$ boson, a neutral spinless fermion, and two distinct 
visons, simultaneously condensed $h c / e$ vortices and skyrmions.

The possibility of a higher $\mathrm{SO}(5)$ symmetry which unifies $d$-wave superconductivity and antiferromagnetism has been suggested for the high- $T_{c}$ cuprates and organic superconductors in Ref. 55. In the $\mathrm{Sr}_{2} \mathrm{RuO}_{4}$ materials, a similar symmetry has been proposed in Ref. 56 which combines $p$-wave superconductivity and ferromagnetism. An effective model for the coupling of quasiparticles to a fluctuating $\mathrm{SO}(5)$ order parameter has been derived in Ref. 57. In this model holons and spinons are not segregated into independent quasiparticles from the very beginning but are naturally combined into composite quasiparticles which transform as spinors of $\mathrm{SO}(5)$. Such spinors are spin doublets and carry charge $e^{58,59}$ There are also neutral fermions which carry no quantum numbers. One can see a striking resemblance between these excitations and the excitations in the phase CBSBNF. This suggests the interesting possibility that the restoration of the $\mathrm{SO}(5)$ symmetry in models with strong quantum fluctuations manifests itself not in the existence of a bicritical point on the phase diagram, but in the appearance of a specific form of quantum number separation of the electrons. A detailed discussion of quantum disordering phenomena in models with $\mathrm{SO}(5)$ symmetry requires a detailed analysis of the nonAbelian Berry's phases involved in the description of $\mathrm{SO}(5)$ spinors and will be presented in subsequent publications.

The states which we have discussed, as well as the more complicated ones alluded to above, have potential application to a variety of materials, including not only the cuprates, ${ }^{22}$ but also $\mathrm{Sr}_{2} \mathrm{RuO}_{4},{ }^{21}$ heavy fermion superconductors, such as $\mathrm{CeIn}_{3} ;{ }^{24}$ and organic superconductors, such as $\kappa-(E T)_{2} \mathrm{Cu}\left[\mathrm{N}(\mathrm{CN})_{2}\right] \mathrm{Cl}^{23}$ All of these compounds have magnetic (in come cases incommensurate) phases in proximity to $p$-wave or $d$-wave superconducting states. It is possible that pressure, chemical substitution, magnetic field, etc., might drive a transition into one of the phases described here in which the magnetism and the superconductivity are disordered by quantum fluctuations.

Ideas presented in this paper should also apply to BoseEinstein condensates of spinor bosons, such as alkali atoms ${ }^{23} \mathrm{Na}$ and ${ }^{87} \mathrm{Ru}$ which have a hyperfine spin $F=1$. For example, when restricted dimensionality or quantum fluctuations destroy the spin ordering we expect to find condensation of pairs of atoms into a global spin singlet state, and when quantum fluctuations in the charge sector destroy the $\mathrm{U}(1)$ phase ordering we can find states characterized by a spin nematic order. Some of these phenomena have been discussed in Ref. 25.

To summarize, we have studied the possibility of fractionalization in systems with ordering tendencies in the charge and spin sectors, including Kondo lattices, $p$-wave superconductors, and systems with simultaneous $d$-wave superconducting and antiferromagnetic fluctuations. In the case of $p$-wave superconductors we find that the rich internal structure of their order parameter allows for the existence of the following quantum disordered phases: a charge $4 e$ singlet superconductor, a spin singlet insulator, and a spin nematic insulator. For both the $p$ wave superconductors and the $d$-wave superconductor/antiferromagnet systems, we find that the quantum disordered phases may have separated quantum numbers, depending on the topological order, which can be characterized by specifying the nature of the finiteenergy $Z_{2}$ visons.

\section{ACKNOWLEDGMENTS}

E.D. and C.N. thank Aspen Center for Physics for its hospitality during the Winter 2000 Conference "50 Years of Condensed Matter Physics," where some parts of this work were initiated. H.Y.K. and Y.B.K. thank ITP, University of California at Santa Barbara, where some parts of this work were performed. C.N., H.Y.K., and Y.B.K. thank Aspen Center for Physics for its hospitality during the summer workshop in 2000. We also thank Aspen Center for Physics for its hospitality during the winter workshop in 2001. Useful discussions with M.P.A. Fisher, E. Fradkin, S. Kivelson, and M. Sigrist are gratefully acknowledged. This work was supported by the Harvard Society of Fellows (E.D.); NSF under grant Nos. DMR-9983544 (C.N.) and DMR-9983783 (Y.B.K.); the Alfred P. Sloan Foundation (C.N. and Y.B.K.); the Department of Energy, supported (in part) by funds provided by the University of California for the conduct of discretionary research by Los Alamos National Laboratory (H.Y.K.). The work of T.S. at the ITP, Santa Barbara, was supported by the NSF under Grant Nos. DMR-97-04005, DMR95-28578, and PHY99-07949.

\section{APPENDIX A: KONDO LATTICE MODEL}

In this appendix, we provide some of the details of the $Z_{2}$ gauge theory reformulation of the Kondo lattice model discussed in Sec. II. Consider the Hamiltonian in Eq. (1). As in the discussion of the pure exchange Hamiltonian, we first replace the spin operator $S_{r}^{-}$by the boson operator $b_{s r}$ $\sim e^{i \varphi_{r}}$. The exchange Hamiltonian takes the form of Eq. (6) and the Kondo coupling takes the form of Eq. (24). The electron hopping term is unaffected. We now change variables to spinon and holon operators as in Eqs. (7), (26), and (27). The terms $H_{t}, H_{k}$, and $H_{e x}$ are now given by Eqs. (29), (25), and (9), respectively. In the presence of the Kondo coupling between the local moments and the conduction electrons, the total ( $z$ component of the) spin at each site is

$$
n_{r}+\frac{1}{2} c_{r}^{\dagger} \sigma^{z} c_{r}
$$

We therefore define the total spinon number

$$
N_{r}^{t o t}=2 n_{r}+c_{r}^{\dagger} \sigma^{z} c_{r} .
$$

Note that $N_{r}^{\text {tot }}$ is conjugate to the phase $\phi_{r}$ of the spinon field. We will work with the operators $\left(z_{r}, N_{r}^{t o t}, \eta_{\uparrow r}, \eta_{\downarrow}\right)$ instead of the original electron and local spin $\vec{S}_{r}$ operators. This change of variables, however, introduces some redundancy - the Hilbert space of states on which the holon and spinon fields operate is larger than the physical set of states. This may be seen by noting that with the definition above, the operator $N_{r}^{\text {tot }}$ must satisfy 


$$
N_{r}^{t o t}-c_{r}^{\dagger} \sigma^{z} c_{r}=\text { even }
$$

From the definition of the holons, it follows that $c_{r}^{\dagger} \sigma^{z} c_{r}$ $=\eta_{r}^{\dagger} \sigma^{z} \eta_{r}$. Furthermore $\eta_{r}^{\dagger} \sigma^{z} \eta_{r}$ has the same parity as $\eta_{r}^{\dagger} \eta_{r}$. Thus we have the constraint

$$
N_{r}^{t o t}-\eta_{r}^{\dagger} \eta_{r}=\text { even }
$$

The Hamiltonian needs to be supplemented with this constraint to correctly represent the original model (before the change of variables).

It is useful to rewrite the exchange and Kondo parts of the Hamiltonian as follows:

$$
\begin{aligned}
H_{K}+H_{e x}= & J_{K} \sum_{r}\left(\eta_{r \uparrow}^{\dagger} \eta_{r \downarrow}+\text { H.c. }\right)-J \sum_{\left\langle r r^{\prime}\right\rangle}\left(z_{r}^{2 \dagger} z_{r}^{2}+\text { H.c. }\right) \\
& +\frac{U}{4} \sum_{r}\left(N_{r}-1\right)^{2}-U \sum_{r} N_{r}\left(\eta_{r}^{\dagger} \sigma^{z} \eta_{r}\right) \\
& +\frac{U}{4} \sum_{r}\left(\eta_{r}^{\dagger} \sigma^{z} \eta_{r}\right)^{2} .
\end{aligned}
$$

The last term is an interaction between the holons. Clearly this term cannot affect issues of confinement of the holons with the spinons. We will therefore drop it for the present discussion. The last but one term represents an interaction between the spinon density and the holons. We again expect that such an interaction is also unimportant for issues of the stability of fractionalized phases. We will therefore drop this too.

We may now derive a functional-integral representation of the system, proceeding as in Ref. 7 . The resulting action is

$$
S=S_{\tau}+S_{r}+S_{B} .
$$

Here $S_{\tau}$ represents terms involving coupling along the (imaginary) time direction. This and the Berry phase $S_{B}$ are exactly the same as in Ref. 7 . The spatial part of the action is

$$
\begin{gathered}
S_{r}=S_{I}+S_{K}+S_{I I} \\
S_{I}=-\epsilon \sum_{\left\langle r r^{\prime}\right\rangle} t_{r r^{\prime}}\left[z_{r}^{\dagger} z_{r^{\prime}}+\left(\eta_{r^{\prime} \uparrow}^{\dagger} \eta_{r \uparrow}+\eta_{r \downarrow}^{\dagger} \eta_{r^{\prime} \downarrow}\right)+\text { H.c. }\right] \\
S_{K}=+\epsilon J_{K} \sum_{r}\left(\eta_{r \uparrow}^{\dagger} \eta_{r \downarrow}+\text { c.c. }\right) \\
S_{I I}=-\epsilon J \sum_{\left\langle r r^{\prime}\right\rangle}\left(z_{r}^{2 \dagger} z_{r}^{2}+\text { H.c. }\right)
\end{gathered}
$$

We now combine the terms $S_{I}$ and $S_{I I}$ and rewrite them as

$$
-\epsilon J \sum_{\left\langle r r^{\prime}\right\rangle}\left[z_{r^{\prime}}^{\dagger} z_{r^{\prime}}+\frac{t_{r r^{\prime}}}{2 J}\left(\eta_{r^{\prime} \uparrow}^{\dagger} \eta_{r \uparrow}+\eta_{r \downarrow}^{\dagger} \eta_{r^{\prime} \downarrow}\right)\right]^{2}+\text { H.c. }+\mathcal{O}\left(\eta^{4}\right) \text {. }
$$

The last term is a four-holon interaction which we will ignore on the grounds that it cannot affect issues of fractionalization. It is convenient to further rewrite the expression above as follows:

$$
\begin{aligned}
S_{I}+S_{I I}= & \frac{\epsilon J}{2} \sum_{\left\langle r r^{\prime}\right\rangle}\left[\left(z_{r}^{\dagger} z_{r^{\prime}}+\frac{t_{r r^{\prime}}}{2 J} \eta_{r}^{\dagger} \eta_{r^{\prime}}+\text { H.c. }\right)^{2}\right. \\
& \left.+\left(z_{r}^{\dagger} z_{r^{\prime}}-\frac{t_{r r^{\prime}}}{2 J} \eta_{r}^{\dagger} \eta_{r^{\prime}}-\text { H.c. }\right)^{2}\right] .
\end{aligned}
$$

We may now decouple each of these two terms with a real Hubbard-Stratanovich field to write

$$
\begin{gathered}
e^{-\left(S_{I}+S_{I I}\right)}=\int[\mathcal{D} \chi \mathcal{D} \rho] e^{-\left(S_{\chi}+S_{\rho}\right)}, \\
S_{\chi}=\frac{\epsilon J}{2} \sum_{\left\langle r r^{\prime}\right\rangle} \chi_{r r^{\prime}}^{2}-2 \chi_{r r^{\prime}}\left(z_{r}^{\dagger} z_{r^{\prime}}+\frac{t_{r r^{\prime}}}{2 J} \eta_{r}^{\dagger} \eta_{r^{\prime}}+\text { H.c. }\right), \\
S_{\rho}=\frac{\epsilon J}{2} \sum_{\left\langle r r^{\prime}\right\rangle} \rho_{r r^{\prime}}^{2}-2 \rho_{r r^{\prime}}\left(z_{r}^{\dagger} z_{r^{\prime}}-\frac{t_{r r^{\prime}}}{2 J} \eta_{r}^{\dagger} \eta_{r^{\prime}}-\text { H.c. }\right) .
\end{gathered}
$$

Note that $\chi_{r r^{\prime}}=\chi_{r^{\prime} r}$ while $\rho_{r r^{\prime}}=-\rho_{r^{\prime} r}$. We now consider evaluating the $\chi, \rho$ integrals in a saddle-point approximation. Looking for uniform saddle points, we write

$$
\left\langle\chi_{r r^{\prime}}\right\rangle=\chi_{0} ; \quad\left\langle\rho_{r r^{\prime}}\right\rangle=\rho_{0}
$$

Note that a nonzero value of $\rho_{0}$ requires specifying directions for all the links of the lattice. The saddle-point equations are

$$
\begin{aligned}
& \chi_{0}=\left\langle z_{r}^{\dagger} z_{r^{\prime}}+\frac{t_{r r^{\prime}}}{2 J} \eta_{r}^{\dagger} \eta_{r^{\prime}}+\text { H.c. }\right\rangle, \\
& \rho_{0}=\left\langle z_{r}^{\dagger} z_{r^{\prime}}-\frac{t_{r r^{\prime}}}{2 J} \eta_{r}^{\dagger} \eta_{r^{\prime}}-\text { H.c. }\right\rangle .
\end{aligned}
$$

Note that $\rho_{0}$ must be pure imaginary as it is the expectation value of an anti-Hermitian operator. With nonzero $\rho_{0}$, the saddle-point action therefore becomes complex - this breaks time-reversal symmetry (and possibly various lattice symmetries due to the need to specify directions to the links). We restrict ourselves to time-reversal invariant saddle-point solutions, and therefore set $\rho_{0}=0$. The resulting saddle-point action then preserves all the global symmetries of the original model. However, it does break the local $Z_{2}$ symmetry introduced by the change of variables to the holons and the spinons. This can be remedied by keeping a particular set of fluctuations about the saddle point, namely those associated with a change in the sign of the fields $\chi_{r r^{\prime}}$ :

$$
\chi_{r r^{\prime}}=\chi_{0} \sigma_{r r^{\prime}}
$$

with $\sigma_{r r^{\prime}}= \pm 1$. The $\sigma_{r r^{\prime}}$ may be identified as the spatial components of a $Z_{2}$ gauge field. We thus finally arrive at the action in Eq. (30). 


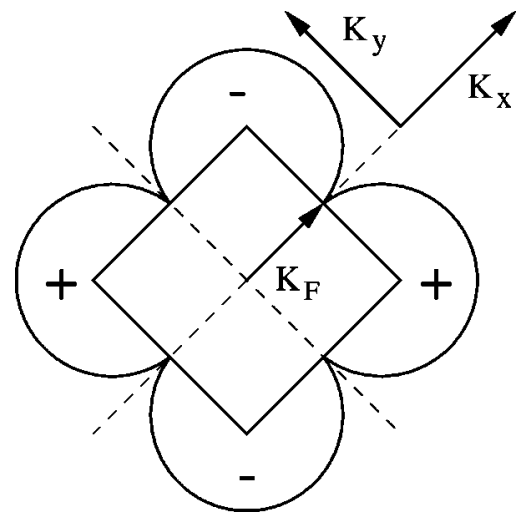

FIG. 7. Order parameter for a $d$-wave superconductor. Gapless excitations exist at $\vec{k}_{F}=\left( \pm k_{F}, 0\right),\left(0, \pm k_{F}\right)$.

\section{APPENDIX B: QUANTUM NUMBER SEPARATION IN SYSTEMS WITH $d$-WAVE SUPERCONDUCTING AND ANTIFERROMAGNETIC FLUCTUATIONS} by $^{28}$

Nodal fermions in a $d$-wave superconductor are described

$$
\begin{aligned}
\widetilde{S}_{f}= & \int d^{2} x d \tau \chi^{\dagger}\left[\partial_{\tau}-A_{\tau} \tau^{z}-v_{F} \tau^{z} i \partial_{x}+v_{F} A_{x}-A_{\tau}^{\sigma} \vec{n} \vec{\sigma}\right. \\
& \left.+v_{f} A_{x}^{\sigma} \vec{n} \vec{\sigma} \tau_{z}-v_{\Delta} \tau^{s}\left\{e^{i s \varphi}\right\}\left(i \partial_{y}\right)\right] \chi,
\end{aligned}
$$

where the electron operators $\chi_{a \alpha}$ are defined as

$$
\chi_{a \alpha}(\vec{k})=\left[\begin{array}{c}
\chi_{11} \\
\chi_{21} \\
\chi_{12} \\
\chi_{22}
\end{array}\right]=\left[\begin{array}{c}
c_{\vec{k}_{F}+\vec{k} \uparrow} \\
c_{-\vec{k}_{F}-\vec{k} \downarrow}^{\dagger} \\
c_{\vec{k}_{F}+\vec{k} \downarrow} \\
-c_{-\vec{k}_{F}-\vec{k} \uparrow}^{\dagger}
\end{array}\right]
$$

and the coordinate system was rotated in such a way that the $x$ axis goes along the nodal direction that we are considering (see Fig. 7).

Antiferromagnetic fluctuations are introduced via

$$
\begin{aligned}
S_{a f} & =\int d \tau \int d^{2} k d^{2} q \vec{n}_{q} c_{-\vec{k}_{F}+\vec{k} \alpha}^{\dagger} \vec{\sigma}_{\alpha \beta} c_{\vec{k}_{F}+\vec{k}+\vec{q} \beta}+\text { H.c. } \\
& =\int d^{2} x d \tau \vec{n}(x) \chi_{a \alpha}(x) \epsilon_{a b} \epsilon_{\alpha}^{\gamma} \vec{\sigma}_{\gamma \beta} \chi_{b \beta}(x),
\end{aligned}
$$

where $\vec{n}=(\cos \theta, \sin \theta)$.

Spin and charge may again be decoupled by rotating the fermions as in Eq. (48),

$$
\chi_{a \alpha}=e^{i \varphi \tau^{z} / 2} e^{i \theta \sigma^{z} / 2} \psi_{a \beta}
$$

with the result

$$
\begin{aligned}
\widetilde{S}= & \int d^{2} x d \tau \psi^{\dagger}\left[\partial_{\tau}-v_{F} \tau^{z} i \partial_{x}-v_{\Delta} \tau^{x}\left(i \partial_{y}\right)\right] \psi \\
& +\int d^{2} x d \tau \psi_{a \alpha}(x) \epsilon_{a b} \epsilon_{\alpha}^{\gamma} \sigma_{\gamma \beta}^{x} \psi_{b \beta}(x) \\
& +\frac{1}{2} \int d^{2} x d \tau\left(\psi^{\dagger}\left[\tau^{z} \partial_{\tau} \varphi-2 A_{\tau}^{c} \tau^{z}-v_{F} \partial_{x} \varphi+2 v_{F} A_{x}^{c}\right] \psi\right. \\
& \left.+\psi^{\dagger}\left[\sigma^{z} \partial_{\tau} \theta-2 A_{\tau}^{\sigma} \tau^{z}-v_{F} \tau^{z} \sigma^{z} \partial_{x} \theta+2 v_{F} \tau^{z} \sigma^{z} A_{x}^{\sigma}\right] \psi\right) .
\end{aligned}
$$

This describes the same coupling of the quasiparticle currents to the fluctuations of charge and spin as in Eq. (50),

$$
\begin{aligned}
\widetilde{S}_{f}= & \widetilde{S}_{f}^{0}+\int d^{2} x d \tau\left[J_{0}^{c}\left(\partial_{\tau} \varphi-A_{\tau}\right)+J_{x}^{c}\left(\partial_{x} \varphi-A_{x}\right)\right. \\
& \left.+J_{0}^{\sigma}\left(z^{\dagger} \partial_{\tau} z-A_{\tau}^{\sigma}\right)+J_{x}^{\sigma}\left(z^{\dagger} \partial_{x} z-A_{x}^{\sigma}\right)\right]
\end{aligned}
$$

with

$$
\begin{gathered}
J_{0}^{c}=\psi^{\dagger} \tau^{z} \psi, \quad J_{x}^{c}=-v_{F} \psi^{\dagger} \psi, \\
J_{0}^{\sigma}=\psi^{\dagger} \sigma^{z} \psi, \quad J_{x}^{\sigma}=-v_{F} \psi^{\dagger} \sigma^{z} \tau^{z} \psi .
\end{gathered}
$$

Quantum disordering of the superconducting and antiferromagnetic orders in Eq. (B6) may now be achieved by condensing vortices and merons with the possibility of five phases similar to phases $3 \mathrm{~A}-3 \mathrm{E}$ in Sec. IV D:

(A) Spinons and holons confined. No quantum number separation.

(B) Spinons unbound and holons glued to fermions.

(C) Holons free and spinons bound to fermions.

(D) Spinons and holons bound together, decoupled from fermions.

(E) All excitations decoupled. Free holons, spinons, neutral fermions.

\section{APPENDIX C: ADIABATIC CONTINUATION BETWEEN DIFFERENT PHASES OF A $Z_{2} \times Z_{2}$ GAUGE MODEL WITH MATTER FIELDS.}

In the pure $Z_{2} \times Z_{2}$ gauge theory there are five phases that are distinct and separated by phase transitions. A question that we address in this section is whether this distinction survives in the presence of matter fields.

\section{Toy models}

Let us begin with a simple model,

$$
S=-K_{1} \sum_{\square} \prod_{\square} \sigma_{i j}-K_{2} \sum_{\square} \prod_{\square} \tau_{i j}-\beta \sum_{i j} \sigma_{i j} \tau_{i j} v_{i} v_{j},
$$

where $v_{i}= \pm 1$ is an Ising matter field. To construct the full phase digram of this model we consider several limiting cases. When $\beta=0$ we have two independent gauge fields, each of which has confining and deconfining phases. When both $K_{1}$ and $K_{2}$ are small we have a confining phase for both $\sigma$ and $\tau$, that has no extra degeneracy on topologically non- 

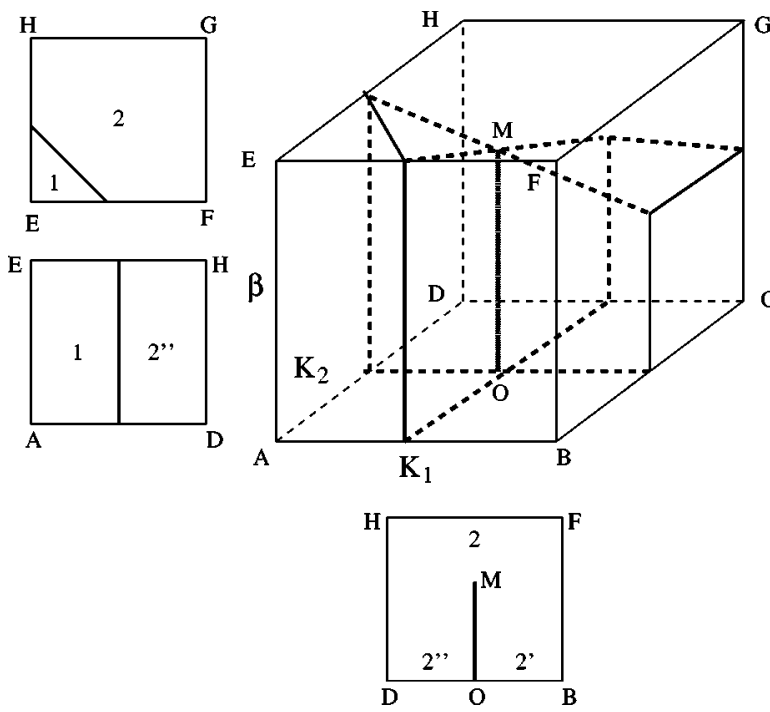
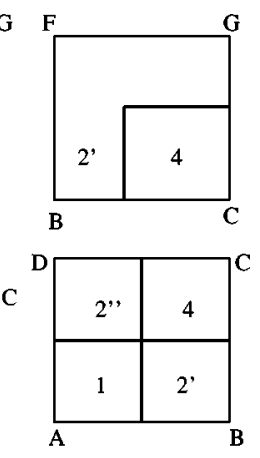

FIG. 8. Phase diagram of Eq. (C1). Plane $\mathrm{ABCD}$ corresponds to $\beta=0, \mathrm{EFGH}$ to $\beta=\infty$, ADHE to $K_{1}=0$, BCFG to $K_{1}=\infty, \mathrm{ABFE}$ to $K_{2}$ $=0$, and DCGH to $K_{2}=\infty$. The pure gauge model $(\beta=0)$ has only two partially confining phases $\left(2^{\prime}\right.$ and $\left.2^{\prime \prime}\right)$ because not all the possible lattice Maxwell terms are present. They are separated by one first-order or two second-order phase transitions for small $\beta$, but may be continuously connected for larger $\beta$.

trivial manifolds and is labeled 1 in Fig. 8. When $K_{1}$ is large and $K_{2}$ is small we have a phase that is confining for $\tau$, but deconfining for $\sigma$ (phase $2^{\prime}$ in Fig. 8). There is an analogous phase for $K_{2}$ large and $K_{1}$ small (phase $2^{\prime \prime}$ in Fig. 8) which is confining for $\sigma$ and deconfining for $\tau$. When both $K$ 's are large, we have a fully deconfining phase with degeneracy 4 on a cylinder. When $K_{1}=\infty$ (BCGF plane in Fig. 8) there are no frustrated plaquettes for $\sigma$, so we can choose a gauge where all $\sigma_{i j}=1$. The model is then the same as in Ref. 46 and its phase diagram can be easily constructed. When $K_{1}$ $=0$ (ADHE plane in Fig. 8) we find that integrating out $\sigma_{i j}$ and $v_{i}$ only adds a constant to the action for $\tau$ and does not affect the confinement-deconfinement transition which takes place for the same value of $K_{2}$, regardless of the value of $\beta$. When $\beta=\infty$ (EFGH plane in Fig. 8) we must have $\Pi_{\square} \sigma_{i j} \tau_{i j}=1$ on every plaquette, so we can choose a gauge where $\sigma_{i j} \tau_{i j}=1$ on every link. The fields $\sigma$ and $\tau$ are identical and there are only two phases, a confining (phase 1) and a deconfining (phase 2) with the transition determined by $K_{1}+K_{2}$. The full phase diagram may now be obtained by connecting the lines on the faces of the cube in Fig. 8. It is immediately clear from this picture that the two partially confining phases which appeared to be distinct for $\beta=0$ (phases $2^{\prime}$ and $2^{\prime \prime}$ in the ABCD plane) may be continuously connected through a path that takes advantage of the finite $\beta$ region of the phase diagram. It is important to realize that our argument for the existence of a path connecting phases 2 ' and $2^{\prime \prime}$ does not depend on the details of how the phase boundaries in Fig. 8 are connected. One can always find a path which begins in phase $2^{\prime}$, approaches face ABFE, goes up to EFGH, crosses to EHDA, and finally comes down to 2 " without crossing the phase boundaries (this path does not have to actually be on any of the faces and it may be sufficient to be in their vicinity). It is interesting to note that in the cross section DBFH in Fig. 8 the phase diagram looks similar to a liquid-gas phase diagram, where the two phases $2^{\prime}$ and $2^{\prime \prime}$ may be separated by a first-order transition or continuously connected arond the critical point which terminates the first-order line.

The real reason why phases $2^{\prime}$ and $2^{\prime \prime}$ of the gauge theory (C1) may be connected to each other is that both of them are related to the Higgs phase (phase 2) for the $v_{i}$ matter field. This may be explained by noting that in such a Higgs phase visons of either $\sigma$ or $\tau$ are forbidden, but their composite is
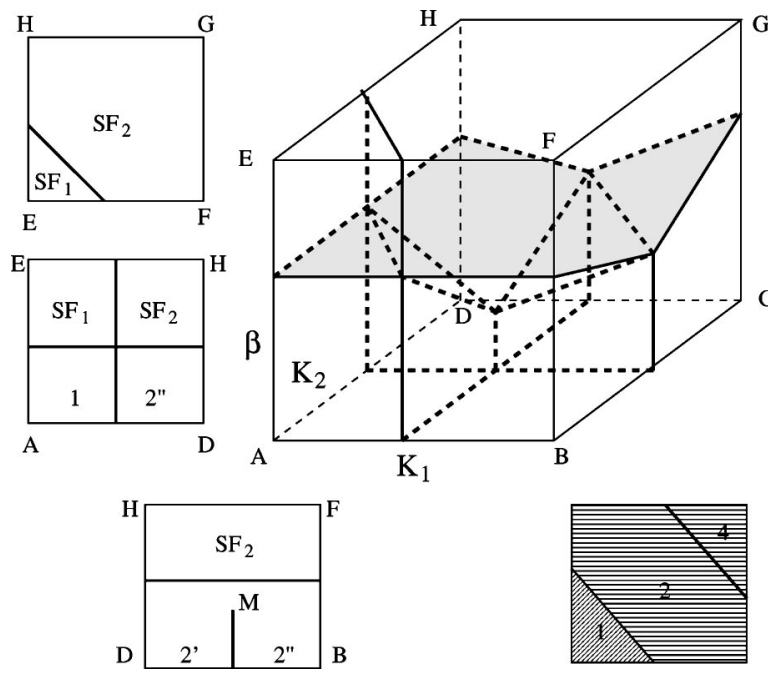
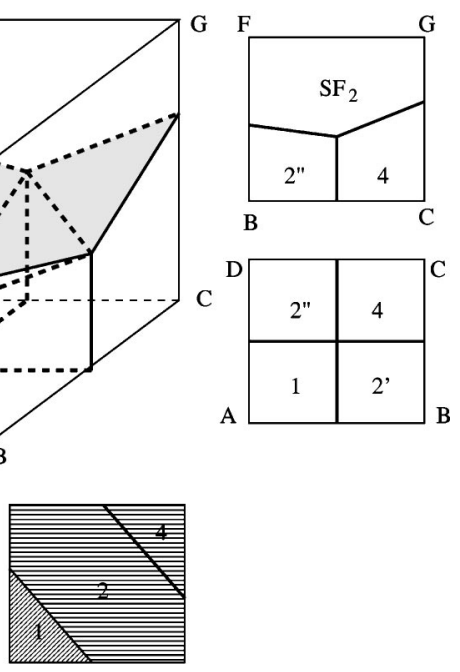

FIG. 9. One scenario for the phase diagram of Eq. (C2) with two kinds of superfluid phases $\mathrm{SF}_{1}$ and $\mathrm{SF}_{2}$. Shaded figure shows a phase boundary of the superfluid and insulating phases. Diagonal shading corresponds to the boundary of $\mathrm{SF}_{1}$ and horizontal shading to a boundary of $\mathrm{SF}_{2}$. There is a continuous path to go between partially confining phases $2^{\prime}$ and $2^{\prime \prime}$ without crossing the phase boundaries. 


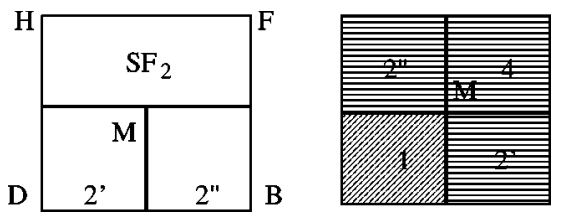

FIG. 10. Another scenario for a phase diagram of Eq. (C2) when the point $M$ is exactly on the superfluid-insulator phase boundary. As before, the shaded figure shows a phase boundary of the superfluid and insulating phases with diagonal and horizontal striping that correspond to $\mathrm{SF}_{1}$ and $\mathrm{SF}_{2}$, respectively. In this case there is no continuous path connecting phases $2^{\prime}$ and $2^{\prime \prime}$ without crossing the phase boundaries.

not, so this phase should be related to the phases where these visons are condensed separately (but not simultaneously).

Note that this argument may no longer apply if the matter field carries a quantum number, and a Higgs phase breaks some continuous symmetry. Let us, for example, explore the model where the matter field is an $X Y$ order parameter,

$$
\begin{aligned}
S= & -K_{1} \sum_{\square} \prod_{\square} \sigma_{i j}-K_{2} \sum_{\square} \prod_{\square} \tau_{i j} \\
& -\beta \sum_{i j} \sigma_{i j} \tau_{i j} \cos \left(\phi_{i}-\phi_{j}\right) .
\end{aligned}
$$

In this case, the Higgs phase has superfluid order, and therefore is fundamentally distinct from confined insulating phases. Thus we can no longer easily claim the equivalence of the two phases in which either $\sigma$ or $\tau$ (though not both) fields are confining.

We can again attempt to construct a phase diagram following construction on each of the outside faces of the cube. The ABCD plane is the same as in Fig 8. The BCGF plane $\left(K_{1}=\infty\right)$ will now have three phases: a confining and a deconfining phases without broken $X Y$ symmetry (phases $2^{\prime \prime}$ and 4), and a phase with broken $X Y$ symmetry. ${ }^{60}$ When $K_{1}$ $=0$ (ADEH plane) we have four phases. This is obvious from the fact that when we integrate out $\sigma_{i j}$ we find that $\tau_{i j}$ and $\cos \phi_{i}$ are decoupled from each other, and we have separate order-disorder and confinement-deconfinement transitions. We therefore find two superfluid phases $\mathrm{SF}_{1}$ and $\mathrm{SF}_{2}$ that differ in their degeneracy on the nontrivial manifolds (on the cylinder it is 1 for $\mathrm{SF}_{1}$ and 2 for $\mathrm{SF}_{2}$ ). The origin of this extra degeneracy for $\mathrm{SF}_{2}$ is that it has a finite energy topo- logical excitation: a bound state of $\sigma$ and $\tau$ visons that does not interact with the matter field. This has interesting implication that we have $h c / 2 e$ vortices that are bound to either $\sigma$ or $\tau$ visons, and the two kinds of vortices are distinct.

We do not at this stage know what the generic phase diagram for Eq. (C2) in the $K_{1} K_{2} \beta$ cube is. One possibility is shown in Fig. 9. As in the Ising case there is a way to connect phases $2^{\prime}$ and $2^{\prime \prime}$ continuously by going to finite $\beta$.

There is another qualitatively different phase diagram (without introducing new phases) where the point $M$ is at the phase boundary with the superfluid phase. This will remove the possibility of a continuous path between phases $2^{\prime}$ and $2 "$ (see Fig. 10).

At this point we are unable to make a definite comment on of the validity of either of the scenarios shown in Fig. 9 or Fig. 10. We note, however, that this issue is amenable to study by numerical or other means. Thus future work should be able to settle this satisfactorily.

Another important model to consider is one in which the matter field is Fermionic. An appropriate model is

$$
S=-K_{1} \sum_{\square} \prod_{\square} \sigma_{i j}-K_{2} \sum_{\square} \prod_{\square} \tau_{i j}-\beta \sum_{i j} \sigma_{i j} \tau_{i j} \psi_{i} \psi_{j},
$$

where the $\psi$ 's are real fermions. Following the same kind of arguments as before we find the phase diagram shown in Fig. 11. There is no Higgs phase for the fermions which leads to phases $2^{\prime}$ and $2^{\prime \prime}$ being distinct even for finite $\beta$.

\section{Full Action}

Let us now consider the action (105) and ask how many truly distinct phases it has. The phase space of this model is large and an explicit construction of the full phase diagram is difficult. We note, however, that the charge sector of the theory is precisely the same as Eq. (C2). Consequently, if in Eq. C2, the two phases 2 and $2^{\prime}$ are smoothly connected to each other, they will necessarily be so for the full action as well. If on the other hand, in Eq. (C2), the two phases are distinct, then that is evidence (though not proof) that they are distinct in the full theory as well. Thus unambiguous determination of the phase diagram of Eq. (C2) will shed considerable light on the important conceptual issue of whether CBSF and CFSB are distinct or not.
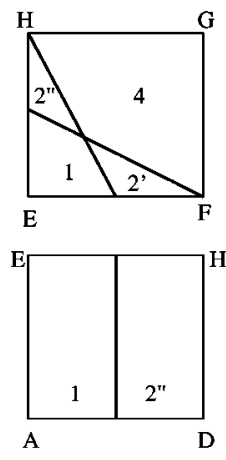

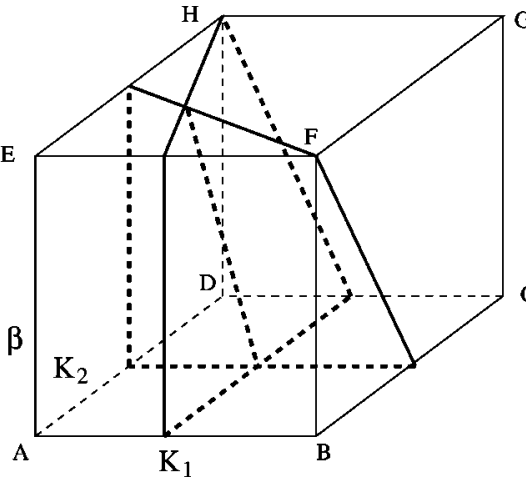

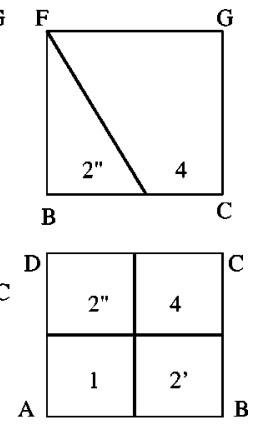

FIG. 11. Phase diagram of Eq. (C3). When the matter field is Fermionic distinction between phases $2^{\prime}$ and $2^{\prime \prime}$ survives for all $\beta$. 
While the distinction between CBSF and CFSB, if any, is subtle, it is very clear that they are both distinct from CSBNF. One cannot find any vison attachment that would map the spectrum of CSBNF to either CBSF or CFSB, which proves rigorously that it is a phase fundamentally distinct from the other two.

To summarize the discussion in this section, we argued that studies, numerical or otherwise, of simple models of the form of Eq. (C2) should be extremely useful in deciding on the issue of whether CBSF and CFSB are distinct quantum phases. One can, however, prove rigorously that CBSF and CFSB are fundmentally different from the other partially confining phase of Eq. (105) CSBNF. The other phases of Eq. (105): the fully confining phase (CSF) and the fully deconfining phase (CBSBNF), will be distinct from any of the partially confining ones and from each other as may be seen from their degeneracy on nontrivial manifolds.

\section{APPENDIX D: UNFRACTIONALIZED PHASES}

In this paper, we have, for the most part, focused on states in which the electron is fractionalized. However, even the transitions which do not lead to electron fractionalization are rather interesting. One would ordinarily assume that strong quantum fluctuations will completely disorder a $p$-wave superconductor. However, as we pointed out in Sec. IV B, if $h c / 4 e$ vortex- $\pi$ disclination composites are gapped, then the spin symmetry can be restored without affecting the charge; alternatively, the superconductivity can be destroyed without affecting the spin ordering.

Let us consider, first, what happens when flux $h c / 2 e$ vortices condense, but no other topological defects condense. Then the charged degrees of freedom are disordered, but the spin nematic order parameter should be undisturbed. Following the arguments of Ref. 61, a possible unfractionalized spin nematic insulating state is (in the notation of Ref. 61) a triplet $p_{x}$ density wave:

$$
\left\langle\psi^{\alpha \dagger}(k+Q, t) \psi_{\beta}(k, t)\right\rangle=\vec{\Phi}_{Q} \cdot \vec{\sigma}_{\beta}^{\alpha} \sin k_{x} a .
$$

This state is related to the $p$-wave superconducting state by a "rotation" generated by

$$
O^{+}=\int \frac{d^{2} k}{(2 \pi)^{2}} c_{\uparrow}^{\dagger}(k) c_{\downarrow}^{\dagger}(-k+Q) .
$$

In other words, the triplet $p_{x}$ density wave and the $p$-wave superconductor are related in precisely the same way as a charge-density wave and an $s$-wave superconductor. In particular, Hamiltonians with short-ranged interactions can be constructed for which both states are exactly degenerate; such Hamiltonians could describe a critical point between these two states.

In the triplet $p_{x}$ density wave state, there is no spin moment (at any wave vector), since the right-hand side of Eq. (D1) vanishes upon integration over $\vec{k}$. However, the spinnematic order parameter, which may be calculated from Eq. (D1), is nonvanishing:

$$
\left\langle S_{i} S_{j}-\frac{1}{3} \delta_{i j} S^{2}\right\rangle=\frac{1}{2}\left|\vec{\Phi}_{Q}\right|^{2} \operatorname{diag}(2 / 3,-1 / 3,-1 / 3),
$$

where $S_{i}$ is the $i$ th component of the total spin of the system. Hence this is the natural spin-nematic state which results when a $p$-wave superconductor is quantum disordered by flux- $h c / 2 e$ vortex condensation. The possibility of spin nematic states in the context of high- $T_{c}$ cuprates has been proposed in Ref. 62.

When the spin degrees of freedom are disordered, but the charge remains ordered, the triplet $p$-wave superconducting order parameter and the spin-nematic order parameter vanishes; only the charge- $4 e$ order parameter is left. The condensation of merons causes the Fermionic quasiparticles to be confined to spin. Once the merons have condensed, the topological quantum number in the spin sector is no longer well defined, so the flux $h c / 4 e$ vortex- $\pi$ disclination composites become simple flux $h c / 4 e$ vortices, as we would expect for a charge- $4 e$ superconductor. Said differently, the meron condensate screens the spin topological charge of the flux $h c / 4 e$ vortex- $\pi$ disclination composites, thereby making rendering them simple flux $h c / 4 e$ vortices. Remarkably, by quantum disordering the spin sector of a $p$-wave superconductor, we have changed the charge of its order parameter, which may, for example, lead to some unusual critical behavior of the superconducting transition.
${ }^{1}$ P.W. Anderson, Science 235, 1196 (1987).

${ }^{2}$ D.S. Rokhsar and S. Kivelson, Phys. Rev. Lett. 61, 2376 (1988).

${ }^{3}$ X.G. Wen, Phys. Rev. B 44, 2664 (1991).

${ }^{4}$ T. Senthil and Matthew P.A. Fisher, Phys. Rev. B 63, 134521 (2001).

${ }^{5}$ D.P. Arovas and A. Auerbach, Phys. Rev. B 38, 316 (1988).

${ }^{6}$ B. Shraiman and E. Siggia, Phys. Rev. Lett. 62, 1564 (1989); C. Jayaprakash, H.R. Krishnamurthy, and S. Sarker, Phys. Rev. B 40, 2610 (1989); K. Feinsberg, P. Hedegård, and M. Brix Pedersen, ibid. 40, 850 (1989); C.L. Kane, P.A. Lee, T.K. Ng, B. Chakraborty, and N. Read, ibid. 41, 2653 (1990).

${ }^{7}$ T. Senthil and Matthew P.A. Fisher, Phys. Rev. B 62, 7850 (2000).

${ }^{8}$ N. Read and S. Sachdev, Phys. Rev. Lett. 66, 1773 (1991); S.
Sachdev and N. Read, Int. J. Mod. Phys. B 5, 219 (1991).

${ }^{9}$ R. Jalabert and S. Sachdev, Phys. Rev. B 44, 686 (1991).

${ }^{10} \mathrm{~S}$. Sachdev and M. Vojta, J. Phys. Soc. Jpn. 69, Suppl. B, 1 (2000).

${ }^{11}$ X.G. Wen and Q. Niu, Phys. Rev. B 41, 9377 (1990); X.G. Wen, ibid. 44, 2664 (1991).

${ }^{12}$ S.A. Kivelson and D.S. Rokhsar, Phys. Rev. B 41, 11693 (1990).

${ }^{13}$ L. Balents, M.P.A. Fisher, and C. Nayak, Phys. Rev. B 60, 1654 (1999).

${ }^{14}$ P.W. Anderson, Mater. Res. Bull. 8, 153 (1973); P. Fazekas and P.W. Anderson, Philos. Mag. 30, 23 (1974).

${ }^{15}$ S.A. Kivelson, D.S. Rokhsar, and J.P. Sethna, Phys. Rev. B 35, 8865 (1987).

${ }^{16}$ F.D.M. Haldane and H. Levine, Phys. Rev. B 40, 7340 (1989). 
${ }^{17}$ N. Read and B. Chakraborty, Phys. Rev. B 40, 7133 (1989).

${ }^{18}$ S. A. Kivelson, Phys. Rev. B 39, 259 (1989).

${ }^{19}$ T. Senthil and Matthew P.A. Fisher, Phys. Rev. Lett. 86, 292 (2001).

${ }^{20}$ A.J. Leggett, Rev. Mod. Phys. 47, 331 (1975).

${ }^{21}$ Y. Maeno et al., Nature (London) 372, 532 (1994).

${ }^{22}$ J.G. Bednorz and K.A. Muller, Z. Phys. B: Condens. Matter 64, 189 (1986).

${ }^{23}$ R.H. MacKenzie, Science 65, 820 (1997); S. Lefebvre et al., cond-mat/0004455 (unpublished).

${ }^{24}$ G. Stewart, Rev. Mod. Phys. 56, 755 (1984).

${ }^{25}$ E. Demler and F. Zhou, cond-mat/0104409 (unpublished).

${ }^{26}$ See, for example, Brandon Carter, astro-ph/0010109 (unpublished).

${ }^{27}$ T. Senthil and M.P.A. Fisher, J. Phys. A 34, L119 (2001).

${ }^{28}$ L. Balents, M. Fisher, and C. Nayak, Int. J. Mod. Phys. B 12, 1033 (1998).

${ }^{29}$ L. Balents, M. Fisher, and C. Nayak, Phys. Rev. B 61, 6307 (2000).

${ }^{30}$ G. Baskaran, Z. Zou, and P.W. Anderson, Solid State Commun. 63, 973 (1987); J.B. Marston and I. Affleck, Phys. Rev. B 39, 11 538 (1989); G. Baskaran and P.W. Anderson, ibid. 37, 580 (1988); G. Kotliar and Liu Jialin, ibid. 38, 5142 (1988); N. Read and B. Chakraborty, ibid. 40, 7133 (1989); L.B. Ioffe and A.I. Larkin, ibid. 39, 8988 (1989); P.A. Lee and N. Nagaosa, ibid. 46, 5621 (1992); A.M. Tikofsky and R.B. Laughlin, ibid. 50, 10165 (1994); B.L. Altshuler, L.B. Ioffe, and A.J. Millis, ibid. 53, 415 (1996); P.A. Lee and X.G. Wen, J. Phys. Chem. Solids 59, 1723 (1998).

${ }^{31}$ E. Fradkin and S.A. Kivelson, Mod. Phys. Lett. B 4, 225 (1990).

${ }^{32}$ S.L. Sondhi and R. Moessner, Phys. Rev. Lett. 86, 1881 (2000); R. Moessner, S.L. Sondhi, and E. Fradkin, cond-mat/0103396 (unpublished).

${ }^{33}$ C. Nayak and K. Shtengel, cond-mat/0010242, Phys. Rev. B (to be published).

${ }^{34}$ D. Vollhardt and P. Wolfe, The Superfluid Phases of Helium 3 (Taylor and Francis, New York, 1990).

${ }^{35}$ A.M. Polyakov, Gauge Fields and Strings (Harwood Academic Publishers, New York 1987).

${ }^{36}$ A. Auerbach, Interacting Electrons and Quantum Magnetism (Springer-Verlag, New York, 1994).

${ }^{37}$ C. Dasgupta and B. Halperin, Phys. Rev. Lett. 47, 1556 (1981); M.P.A. Fisher and D.-H. Lee, Phys. Rev. B 39, 2756 (1989); M.E. Peskin, Ann. Phys. (N.Y.) 113, 122 (1978); P.R. Thomas and M. Stone, Nucl. Phys. B 144, 513 (1978); X.G. Wen and A.
Zee, Int. J. Mod. Phys. B 4, 437 (1990).

${ }^{38}$ D.H. Lee and C.L. Kane, Phys. Rev. Lett. 64, 1313 (1990).

${ }^{39}$ K. Moon et al., Phys. Rev. B 51, 5138 (1995).

${ }^{40}$ E. Demler, C. Nayak, and S. Das Sarma, Phys. Rev. Lett. 86, 1853 (2001).

${ }^{41}$ S. Sachdev, Phys. Rev. B 49, 6770 (1994).

${ }^{42}$ T.K. Ng, Phys. Rev. Lett. 82, 3504 (1999).

${ }^{43}$ T.K. Ng, cond-mat/9911099 (unpublished).

${ }^{44}$ C. Nayak, Phys. Rev. B 62, R6135 (2000).

${ }^{45}$ In principle, we should also consider nonsinglet charge- $4 e$ superconductors, with $\Delta^{4 e} \neq 0, Q \neq 0$ but vanishing $\Delta_{\alpha \beta}$. These occur when the flux $h c / 4 e$ vortex- $\pi$ disclination composite becomes unstable and the flux $h c / 4 e$ vortex and the $\pi$ disclination exist as separate stable excitations. These states will be discussed in subsequent publications.

${ }^{46}$ E. Fradkin and S.H. Shenker, Phys. Rev. D 19, 3682 (1979).

${ }^{47}$ F. Wegner, J. Math. Phys. 12, 2259 (1971).

${ }^{48}$ Phase Transitions and Critical Phenomena, edited by C. Domb and M. Green (Academic Press, New York, 1977).

${ }^{49}$ X.G. Wen, Adv. Phys. 44, 406 (1995).

${ }^{50}$ A. Fetter et al., Phys. Rev. B 39, 9679 (1989); W. Chen et al., J. Mod. Phys. 3, 1001 (1989); X.G. Wen and A. Zee, Phys. Rev. B 44, 274 (1991).

${ }^{51}$ V. Kalmeyer and R.B. Laughlin, Phys. Rev. Lett. 59, 2095 (1987); X.G. Wen, Phys. Rev. B 40, 7387 (1990); X.G. Wen et al., ibid. 44, 274 (1991).

${ }^{52}$ F. Wilczek, Phys. Rev. Lett. 48, 1144 (1982).

${ }^{53}$ Y.B. Kim, C. Nayak, E. Demler, N. Read, and S. Das Sarma, Phys. Rev. B 63, 205315 (2001).

${ }^{54}$ A.V. Chubukov, S. Sachdev, and T. Senthil, Nucl. Phys. B 426, 601 (1994).

${ }^{55}$ S. C. Zhang, Science 275, 1089 (1997).

${ }^{56}$ S. Murakami, N. Nagaosa, and M. Sigrist, Phys. Rev. Lett. 82, 2939 (1999).

${ }^{57}$ E. Demler and S.-C. Zhang, Ann. Phys. (N.Y.) 271, 83 (1999).

${ }^{58}$ S. Rabello, H. Kohno, E. Demler, and S.-C. Zhang, Phys. Rev. Lett. 80, 3586 (1998).

${ }^{59}$ D.J. Scalapino, S.C. Zhang, and W. Hanke, Phys. Rev. B 58, 443 (1998).

${ }^{60}$ P.E. Lammert, D.S. Rokhsar, and J. Toner, Phys. Rev. Lett. 70, 1650 (1993).

${ }^{61}$ C. Nayak, Phys. Rev. B 62, 4880 (2000).

${ }^{62}$ L.P. Gorkov and A.V. Sokol, JETP Lett. 52, 504 (1990); V. Barzykin, L.P. Gorkov, and A.V. Sokol, Europhys. Lett. 15, 869 (1991). 